\title{
19. SOUTHEAST ATLANTIC DSDP LEG 40 PALEOGENE BENTHIC FORAMINIFERS
}

\author{
Franca Proto Decima, Geological Institute, Padua University, Padua, Italy \\ and \\ Hans M. Bolli, Department of Geology, Swiss Federal Institute of Technology, \\ and University of Zürich, Switzerland
}

\section{INTRODUCTION}

The closely spaced coring of most of the Leg 40 sites offered the opportunity to study Paleogene benthic foraminifers from an area where heretofore almost no information existed. There was also the opportunity to tie in the distribution of the benthic foraminifers with that of the planktonic foraminifers and the calcareous nannoplankton, and thus evaluate their stratigraphic and ecologic significance. Further, it became possible to compare the Cape Basin, Walvis Ridge, and Angola Basin benthic assemblages and to confront them with known age equivalent faunas from other areas, such as New Zealand, the Caribbean, the Gulf Coast, the Caucasus, and the Alpine Mediterranean region, in particular with the recently described fauna of the Possagno section in Northern Italy (Table 10). It was also important to find out whether the Cape Basin fauna shows distinct boreal, Austral/New Zealand province affinities, as do the planktonic foraminifers.

With the studies in this volume on the Neogene benthic foraminifers of Sites 360 and 362 by Cameron, on the Upper Cretaceous of Sites 363 and 364 by Beckmann, and on the Lower Cretaceous of Sites 363 and 364 by Scheibnerová, the inclusion of the Paleogene allows one to trace the benthic foraminifer distribution throughout those sections of all sites where these faunas occurred.

Paleogene benthic foraminifers were investigated from the following Leg 40 sites (Figure 1):

Site 360: Samples 26-1, 136-139 cm (lower Mioceneupper Oligocene to 50, CC (middle Eocene)

Site 361: Samples 1, CC (upper Eocene) to 11, CC (Paleocene)

Site 362A: Samples 2, CC (upper Oligocene to 12-1, $108-110 \mathrm{~cm}$ (lower Eocene)

Site 363: Samples 2, CC (upper Oligocene) to 17, CC (lower Paleocene)

Site 364: Samples 7, CC (middle Eocene) to 10, CC (lower Paleocene)

Much of the preparation for this study was done onboard ship on core-catcher samples. To obtain a more complete faunal picture, one or more additional samples were subsequently selected from each core of Sites 360,363 , and 364 .

Core 9 of Site 363 straddles the Eocene-Oligocene boundary. It therefore received special attention. Across this boundary, a total of eight samples from Section 3 and the upper part of Section 4 were investigated to determine exactly the boundary on the basis of planktonic foraminifers. The boundary lies in

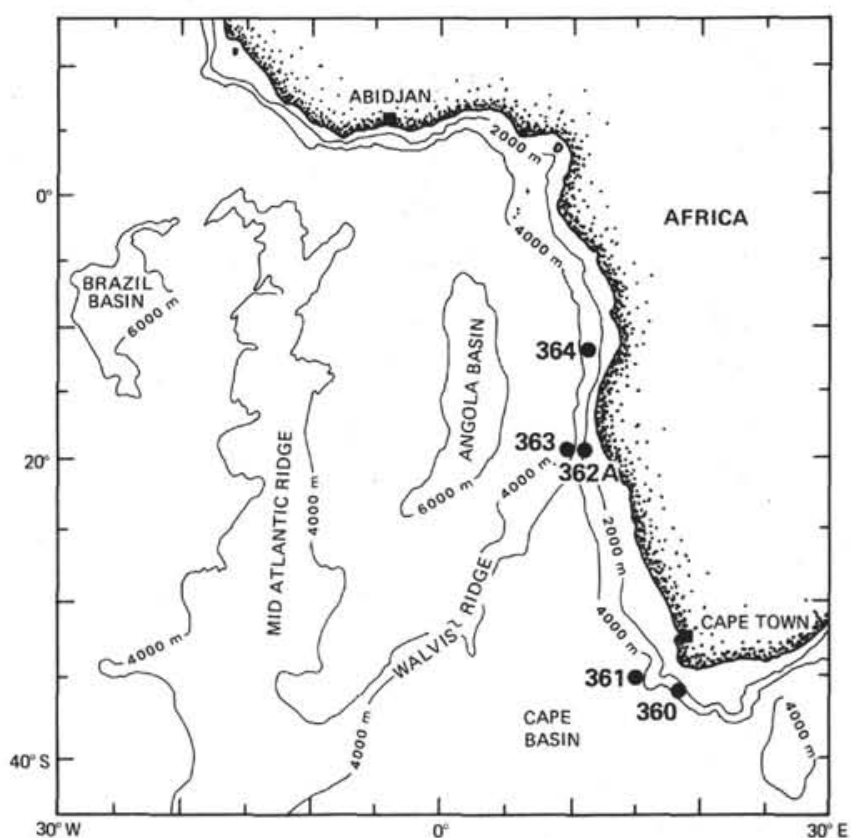

Figure 1. Location of Leg 40 Sites 360-364.

Section 3 between 84 and $98 \mathrm{~cm}$. The benthic foraminifers on this restricted interval (Section 3, and the upper part of Section 4 representing some $2.5 \mathrm{~m}$ ) show numerous first and last occurrences and eight species were found restricted to it.

The taxa recognized in Sites 360, 361, 362A, 363, and 364 are plotted on Tables 1-5 where their ranges are also compared with planktonic foraminifer zones and ages. The ranges are also readily compared with the calcareous nannoplankton zones by means of the zonal correlation charts of the site chapters.

Table 6 shows the species present in all three basins, and Tables 7, 8, and 9 those restricted to the Cape Basin, Walvis Ridge, and Angola Basin, respectively. Tables 10 and 12 plot the species present in the Paleocene and those restricted to the Eocene and Oligocene.

A considerable amount of Paleogene sediments in the Leg 40 sites have been more or less strongly affected by calcium carbonate dissolution. How it affected the planktonic foraminifers at Sites 360, 361, 362A, 363, and 364 is shown by Toumarkine (this volume) on her fig. $2,4,6,8$, and 10 .

It may be assumed that as bottom living forms the tests of the benthic foraminifers were not subject to 
TABLE 1

Distribution of Lower Miocene to Middle Eocene Benthic Foraminifers in Site 360

\begin{tabular}{|c|c|c|c|c|c|c|c|c|c|c|c|c|c|c|c|c|c|c|c|c|c|c|c|c|c|c|c|c|c|c|c|c|c|c|c|c|}
\hline & & 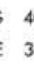 & & & & & & & & & & & & & & & & & & & & & & & & & & & & & & & & & & \\
\hline 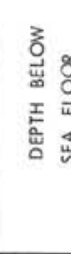 & 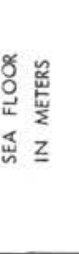 & $\vec{g}$ & & 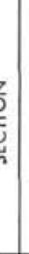 & $\begin{array}{l}\underline{\widetilde{E}} \\
\vec{a} \\
\underline{\underline{z}} \\
\underline{\underline{\underline{z}}}\end{array}$ & 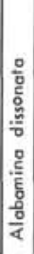 & 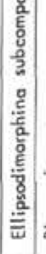 & 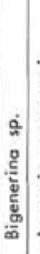 & 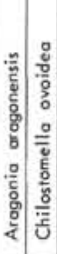 & 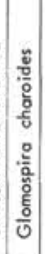 & 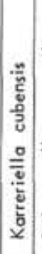 & 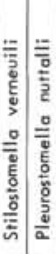 & 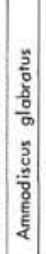 & 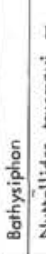 & 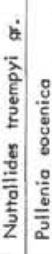 & 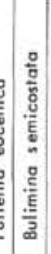 & 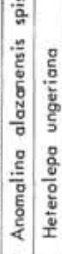 & 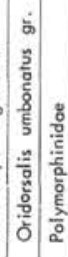 & 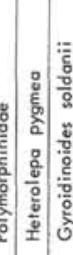 & 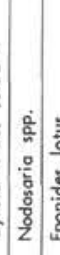 & 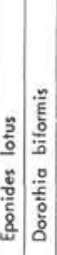 & 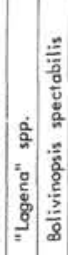 & 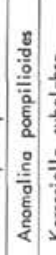 & 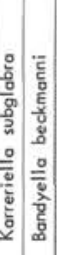 & 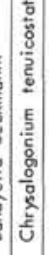 & 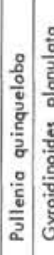 & 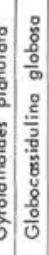 & 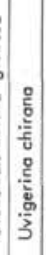 & 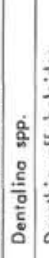 & 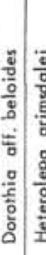 & 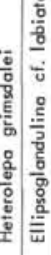 & 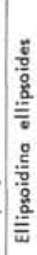 & 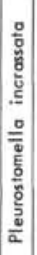 & 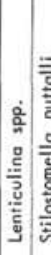 & 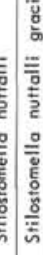 & 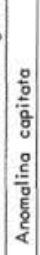 \\
\hline \multirow{2}{*}{\multicolumn{2}{|c|}{$393-402,5$}} & 2 & 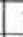 & 1 & $136-139$ & & & & & & & & & & & & & & & & & & & & & & & & & & & & & & & \\
\hline & & & & & $\mathrm{CC}$ & & & & & & & & & & & & & & & & & & & & & & & & & & & & & & & \\
\hline \multirow{2}{*}{\multicolumn{2}{|c|}{$412-421,5$}} & 27 & 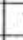 & 1 & $56-58$ & & & & & & & & & & & & & & & & & & & & & & & & & & & & & & & \\
\hline & & & & & $\mathrm{CC}$ & & & & & & & & & & & & & & & & & & & & & & & & & & & & & & & \\
\hline \multirow{2}{*}{\multicolumn{2}{|c|}{$431-440,5$}} & 28 & & 1 & $87-89$ & & & & & & & & & & & & & & & & & & & & & & & & & & & & & & & \\
\hline & & & & 3 & boltom & & & & & & & & & & & & & & & & & & & & & & & & & & & & & & & \\
\hline \multirow{2}{*}{\multicolumn{2}{|c|}{$450-459,5$}} & 29 & & 2 & $73-75$ & & & & & & & & & & & & & & & & & & & & & & & & & & & & & & & \\
\hline & & & & & $C C$ & & & & & & & & & & & & & & & & & & & & & & & & & & & & & & & \\
\hline \multirow[t]{2}{*}{469} & $-478,5$ & 30 & & 3 & $92-94$ & & & & & & & & & & & & & & & & & & & & & & & & & & & & & & & \\
\hline & & & & & $\mathrm{CC}$ & & & & & & & & & & & & & & & & & & & & & & & & & & & & & & & \\
\hline 488 & $-497,5$ & 31 & & & $\mathrm{CC}$ & & & & & & & & & & & & & & & & & & & & & & & & & & & & & & & \\
\hline \multirow{2}{*}{\multicolumn{2}{|c|}{$507-516,5$}} & 32 & & 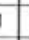 & $107-110$ & & & & & & & & & & & & & & & & & & & & & & & & & & & & & & & \\
\hline & & & & & $\mathrm{CC}$ & & & & & & & & & & & & & & & & & & & & & & & & & & & & & & & \\
\hline \multirow{2}{*}{\multicolumn{2}{|c|}{$526-535,5$}} & 33 & & 3 & $70-22$ & & & & & & & & & & & & & & & & & & & & & & & & & & & & & & & \\
\hline & & & & & $\mathrm{CC}$ & & & & & & & & & & & & & & & & & & & & & & & & & & & & & & & \\
\hline \multirow{2}{*}{\multicolumn{2}{|c|}{$545-554,5$}} & 34 & & 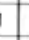 & $89-91$ & & & & & & & & & & & & & & & & & & & & & & & & & & & & & & & \\
\hline & & & & & $C C$ & & & & & & & & & & & & & & & & & & & & & & & & & & & & & & & \\
\hline \multirow[t]{4}{*}{564} & $-573,5$ & 35 & & 2 & $134-136$ & & & & & & & & & & & & & & & & & & & & & & & & & & & & & & & \\
\hline & & & & 3 & $11-14$ & & & & & & & & & & & & & & & & & & & & & & & & & 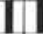 & 1 & & & & & \\
\hline & & & & 3 & $32-33$ & & & & & & & & & & & & & & & & & & & & & & & & & & & & & & & \\
\hline & & & & & $\mathrm{CC}$ & & & & & & & & & & & & & & & & & & & & & & & & & & & & & & & \\
\hline 573,5 & -583 & 36 & & & $C c$ & & & & & & & & & & & & & & & & & & & & & & & & & & & & & & & \\
\hline 592,5 & -602 & 37 & & & $C C$ & & & & & & & & & & & & & & & & & & & & & & & & & & & & & & & \\
\hline 611,5 & -621 & 38 & & & $C C$ & & & & & & & & & & & & & & & & & & & & & & & & & & & & & & & \\
\hline 630,5 & -640 & 39 & & & $C C$ & & & & & & & & & & & & & & & & & & & & & & & & & & & & & & & \\
\hline 649,5 & -659 & 40 & & & $C C$ & & & & & & & & & & & & & & & & & & & & & & & & & & & & & & & \\
\hline 668,5 & -678 & 41 & & & $131-132$ & & & & & & & & & & & & & & & & & & & & & & & & & & & & & & & \\
\hline & & & & & $C c$ & & & & & & & & & & & & & & & & & & & & & & & & & & & & & & & \\
\hline 678 & $-687,5$ & 42 & & & $58-60$ & & & & & & & & & & & & & & & & & & & & & & & & & & & & & & & \\
\hline & & & & & $C C$ & & & & & & & & & & & & & & & & & & & & & & & & & & & & & & & \\
\hline 697 & $-706,5$ & 43 & & & $33-35$ & & & & & & & & & & & & & & & & & & & & & & & & & & & & & & & \\
\hline & & & & & $\mathrm{CC}$ & & & & & & & & & & & & & & & & & & & & & & & & & & & & & & & \\
\hline 716 & $-725,5$ & 44 & & t & $74-76$ & & & & & & & & & & & & & & & & & & & & & & & & & & & & & & 1 & \\
\hline & & & & & $C C$ & & & & & & & & & & & & & & & & & & & & & & & & & & & & & 1 & & \\
\hline 735 & $-744,5$ & 45 & & 3 & $83-85$ & & & & & & & & & & & & & & & & & & & & & & & & & & & & & & & \\
\hline & & & & & $\mathrm{CC}$ & & & & & & & & & & & & & & & & & & & & & & & & & & & & & & & \\
\hline 754 & $-763,5$ & 46 & & & $c c$ & & & & & & & & & & & & & & & & & & & & & & & & & & & & & & & \\
\hline 773 & $-782,5$ & 47 & & & bottom & & & & & & & & & & & & & & & & & & & & & & & & & & & & & & & \\
\hline 792 & $-801,5$ & 48 & & & $\mathrm{CC}$ & & & & & & & & & & & & & & & & & & & & & & & & & & & & & & & \\
\hline 811 & $-820,5$ & 49 & & & $c c$ & & & & & & & & & & & & & & & & & & & & & & & & & & & & & & & \\
\hline 830 & $-839,5$ & 50 & & & $\mathrm{CC}$ & & & & & & & & & & & & & & & & & & & & & & & & & & & & & & & \\
\hline
\end{tabular}

dissolution, but dissolution may have taken place during diagenesis of the sediments.

When evaluating the Paleogene benthic foraminifer species and their ranges presented in this paper, one has to keep in mind that the study is based on a rather limited amount of samples and sediment material.

\section{SOME OBSERVATIONS ON FAUNAL DISTRIBUTIONS}

The benthic foraminifer fauna present in the Paleogene sections of Leg 40 contains species which are already well known from the literature. They are, e.g., recorded from the Lizard Springs and Navet forma- tions of Trinidad, the Velasco Formation of Mexico, the Caucasus, Austria, and Northern Italy, and are thus very similar to those known from the Tethyan regions. Their presence in the southeast Atlantic is further proof for their worldwide distribution and stratigraphic significance.

Faunal assemblages of the kind presented in Leg 40 sediments were termed by Berggren and Aubert (1975) as "Velasco-type." In contrast to the continental shelf "Midway-type," they are deeper water faunas of the lower slope and abyssal plain.

Many of the species listed on Table 6, present in the Cape Basin, on Walvis Ridge, and in the Angola Basin, 
TABLE 1 - Continued

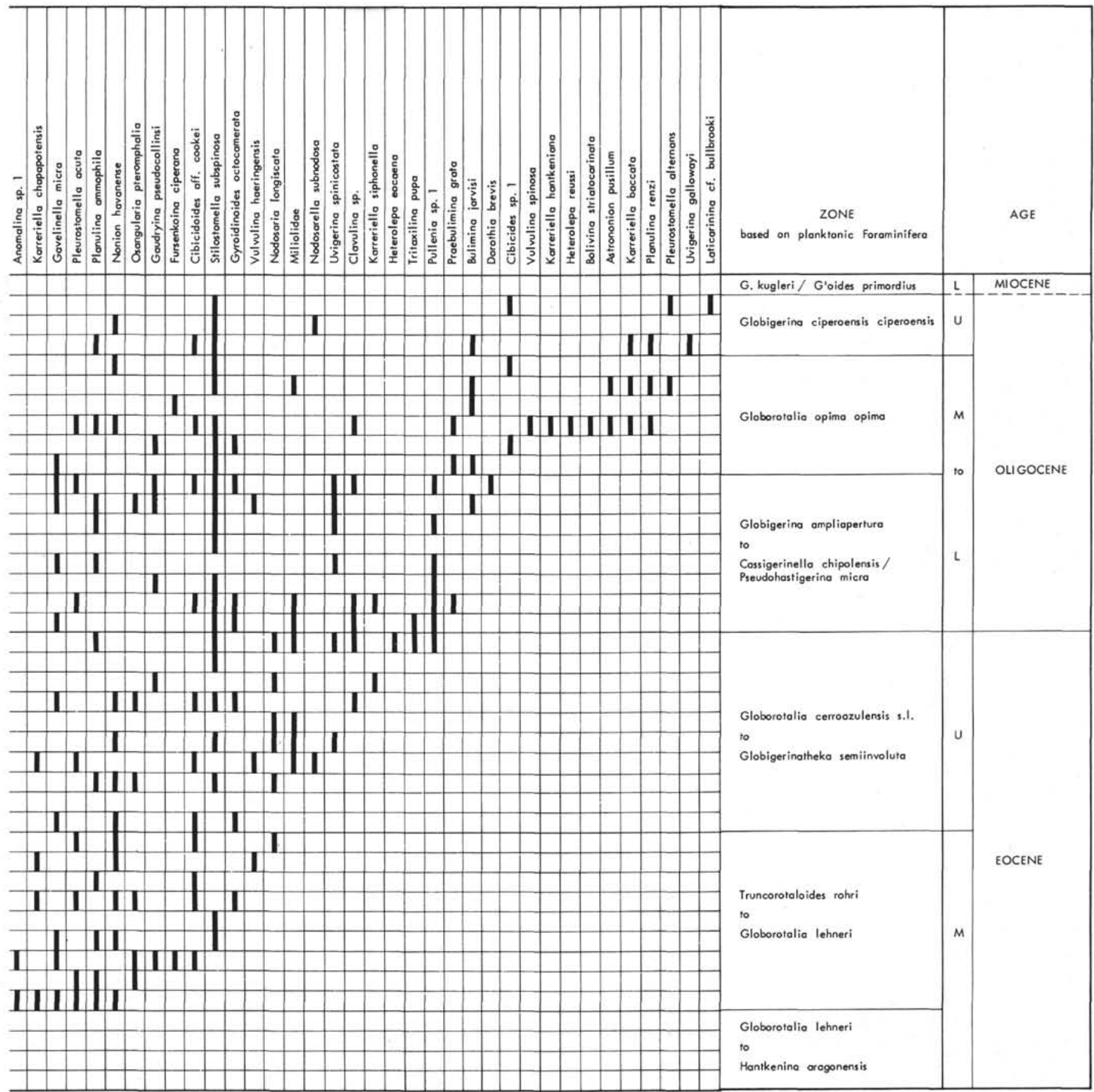

are common forms of no particular stratigraphic value. The extent of their long ranges may vary somewhat from site to site. To them belong Bolivinopsis spectabilis, Gyroidinoides soldanii, Heterolepa ungeriana, Nonion havanense, and Oridorsalis umbonatus 1.s. Others, some of which are mentioned below, have shorter ranges and are considered good index fossils.

Marked differences exist between the Paleocene benthic assemblages of Sites 361, 363, and 364. The discussion below indicates that they are apparently not so much the result of the different latitudes $\left(35^{\circ} \mathrm{S}, 19^{\circ} \mathrm{S}\right.$, and $11^{\circ} \mathrm{S}$ ), but rather of variations in depth and possibly other ecological factors. The greatest number of species was found at Site 363 on Walvis Ridge, where many are restricted to this site. Though this can in part be explained by the greater number of Paleocene samples from this site, we consider shallower water to be a more important factor. Toumarkine (this volume) indicates much better preservation for Site 363 Paleocene planktonic foraminifers than at Sites 361 and 364 . This is probably related to the shallower depth of Site 363 during Paleocene, and in turn allowed for the development of a rich benthic fauna.

The presence here of Neoflabellina jarvisi and $N$. semireticulata and their absence from the other sites, as 
TABLE 2

Distribution of Eocene to Paleocene Benthic Foraminifers in Site 361

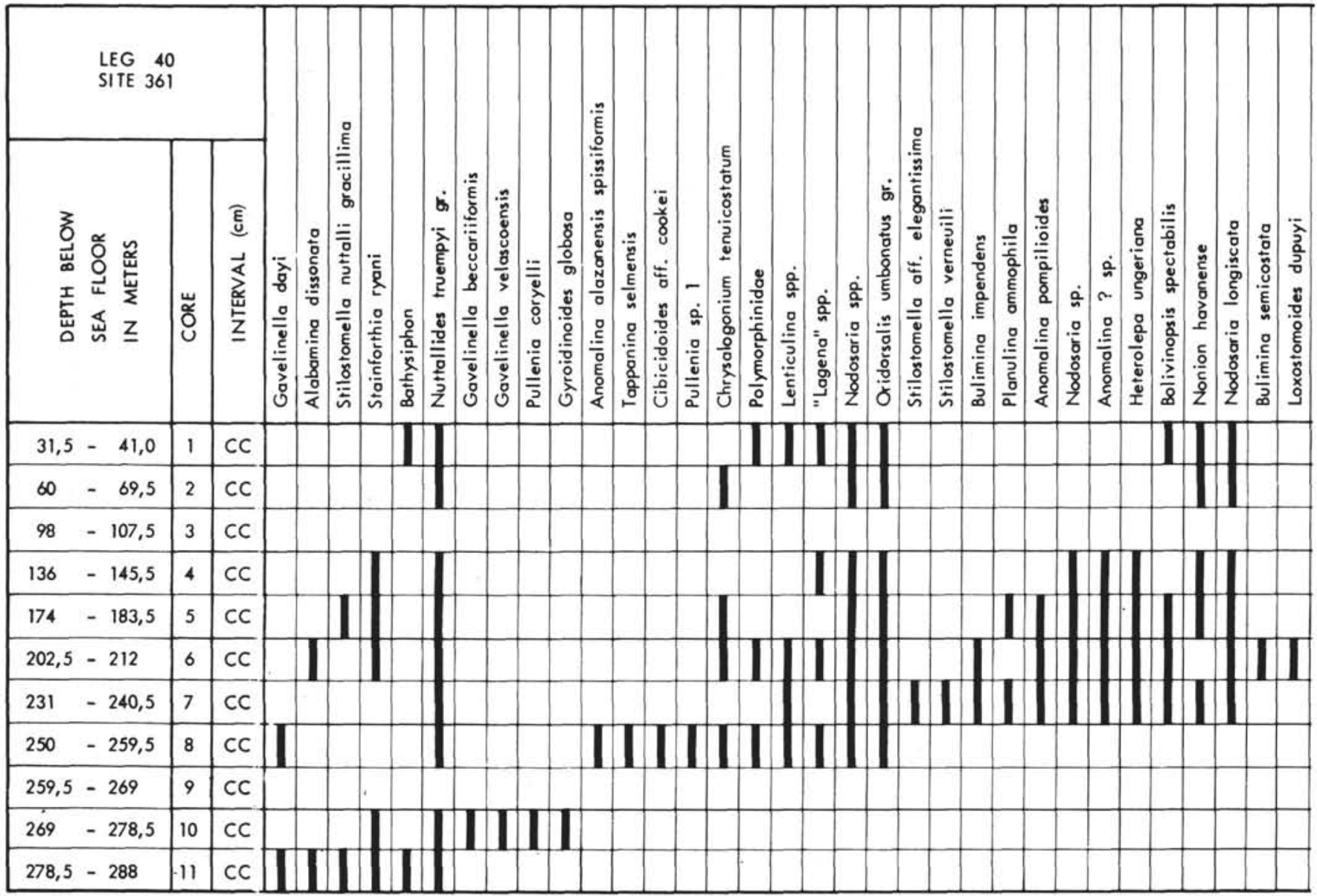

TABLE 3

Distribution of Upper Oligocene to Lower Eocene Benthic Foraminifers in Hole 362A

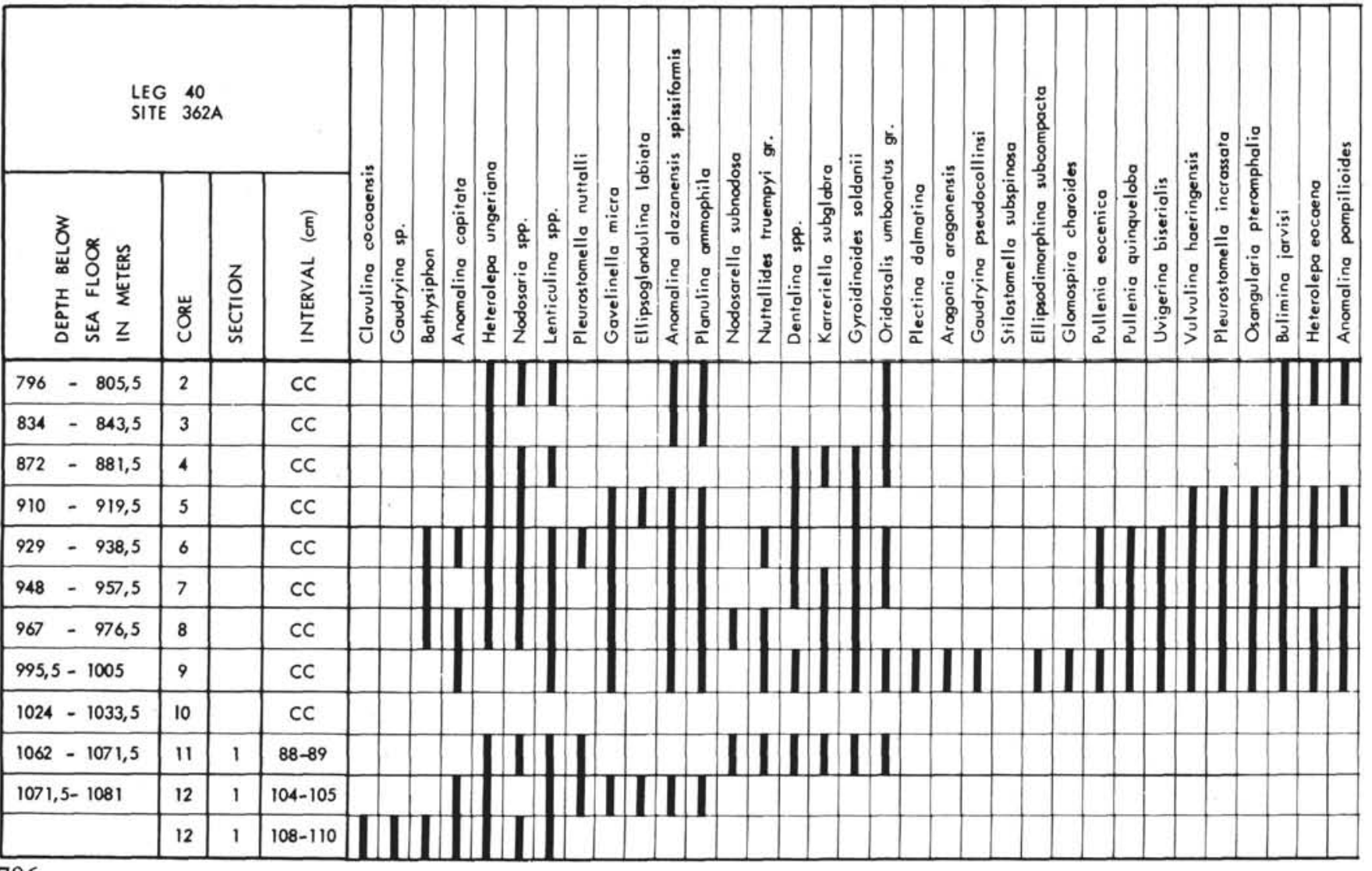


TABLE 2 - Continued

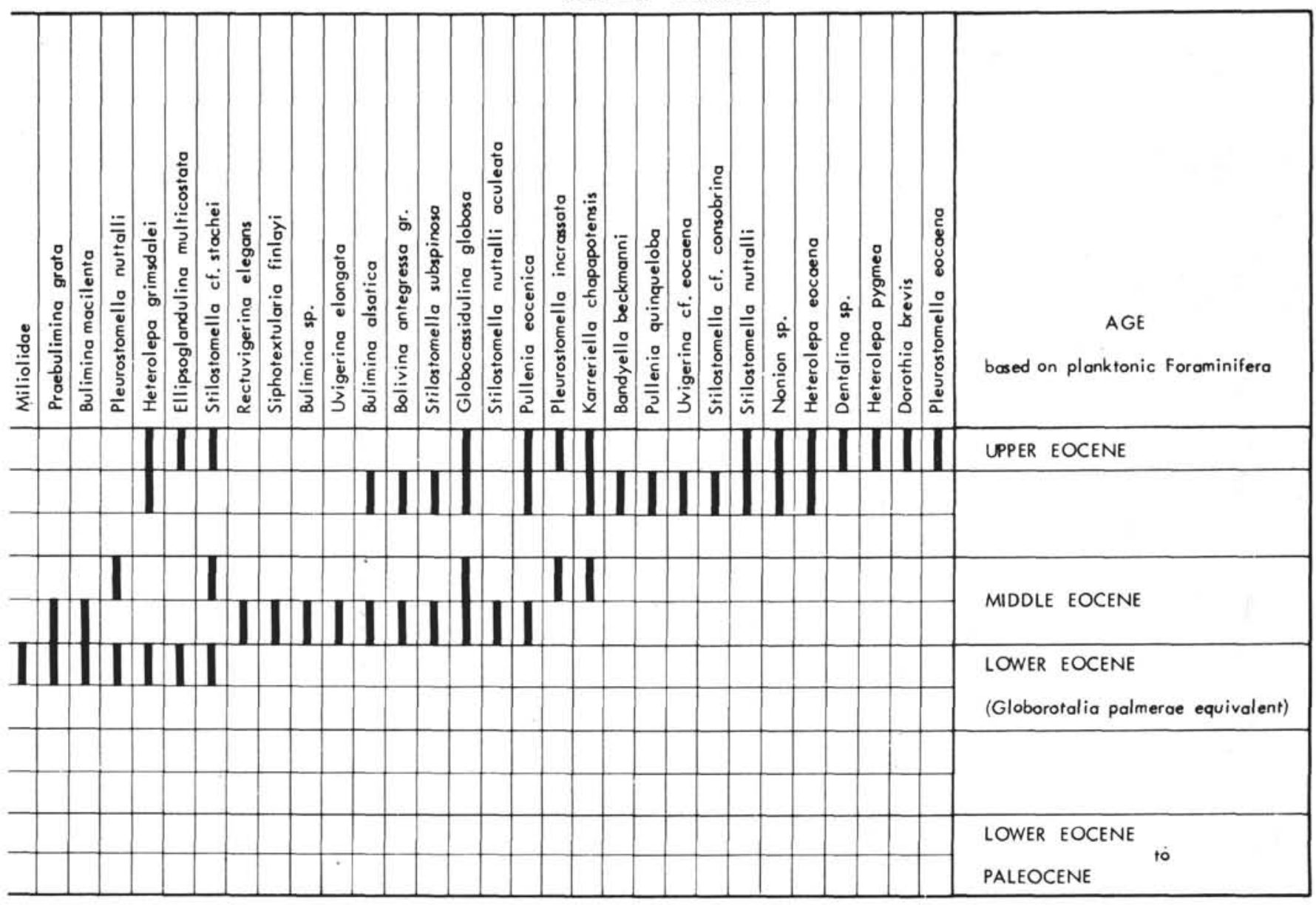

TABLE 3 - Continued

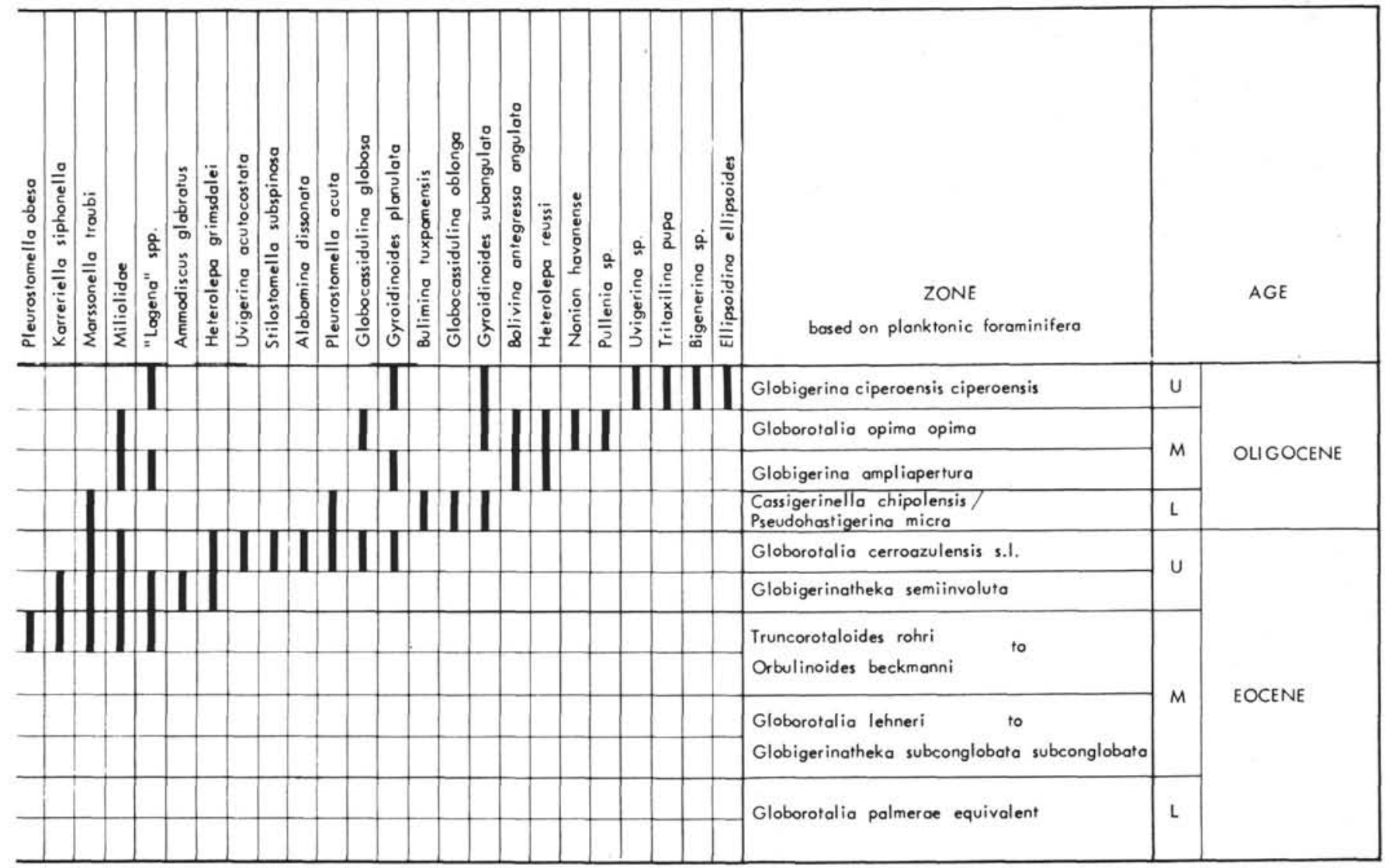


TABLE 4

Distribution of Upper Oligocene to Lower Paleocene Benthic Foraminifers in Site 363

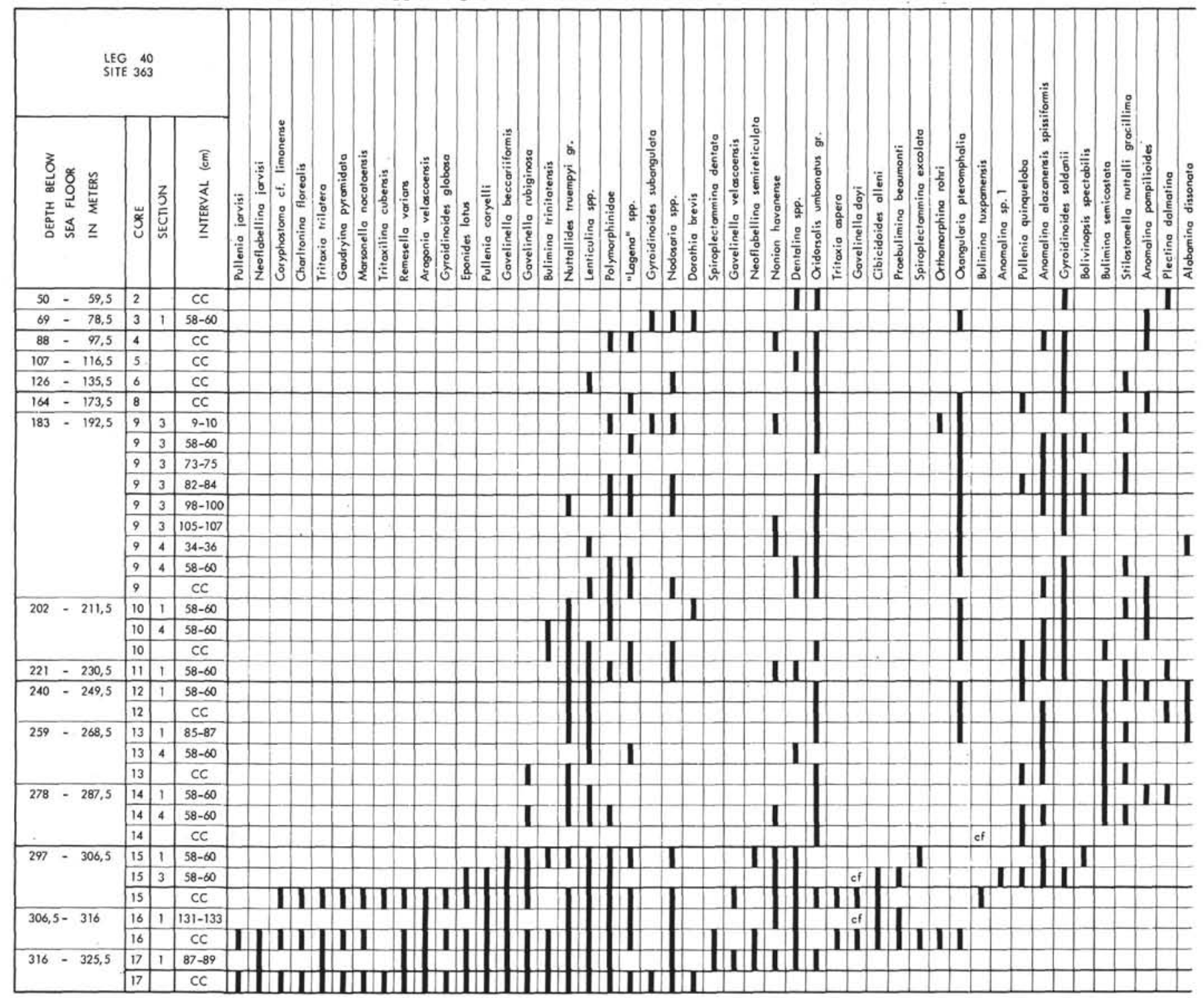

well as the diversity of the higher evolved arenaceous species further support this supposition. The absence of nodosariids and polymorphinids in the Paleocene of Site 361 and the scarce benthic population indicates that Site 361 was deeper than Sites 363 and 364 . The two species Aragonia velascoensis and A. ouezzanensis found in the Paleocene are restricted to Sites 363 and 364 , respectively, and therefore seem to have different ecological distributions.

The Eocene and Oligocene assemblages are less characteristic than those of the Paleocene. They are largely composed of long-ranging species which are of little or no stratigraphic significance. Alabamina dissonata can be considered an Eocene marker since the same range is also recorded in the Caribbean. Its presence in the Paleocene Sample 361-11, CC can be disregarded because this sample contains nannofossil evidence for contamination from above. Other characteristic Eocene species are Uvigerina chirana and Plectina dalmatina, known in the literature also as $P$. eocenica. The first appearance of Heterolepa grimsdalei seems to be a good middle Eocene indicator. Sample
361-6, CC, which also contains this species, is (Table 2) referred to the uppermost lower Eocene Globorotalia palmerae Zone equivalent. However, nannoplankton evidence places this in the Discoaster sublodoensis Zone which could either be uppermost lower or lowermost middle Eocene. The widely known group of Nuttallides truempyi does not cross the Eocene/Oligocene boundary. Of some interest in the Cape Basin Eocene could be the presence of the New Zealand species Dorothia biformis and Siphotextularia finlayi.

Planulina renzi and Karreriella hantkeniana are species restricted to the Oligocene of Leg 40 sites. They can be considered to be good stratigraphic markers in this interval. The first appears in the Globorotalia opima opima Zone of Sites 360 and 363. It is also present in the Caribbean Oligocene, and both species are found in the Oligocene of Northern Italy. Astrononion pusillum, a rare but characteristic species of New Zealand, occurs in the Oligocene of Sites 360 and 363.

Two species are described as new: Stainforthia ryani, which is fairly common in the middle Eocene of the Cape Basin Site 361 and Bandyella beckmanni which 


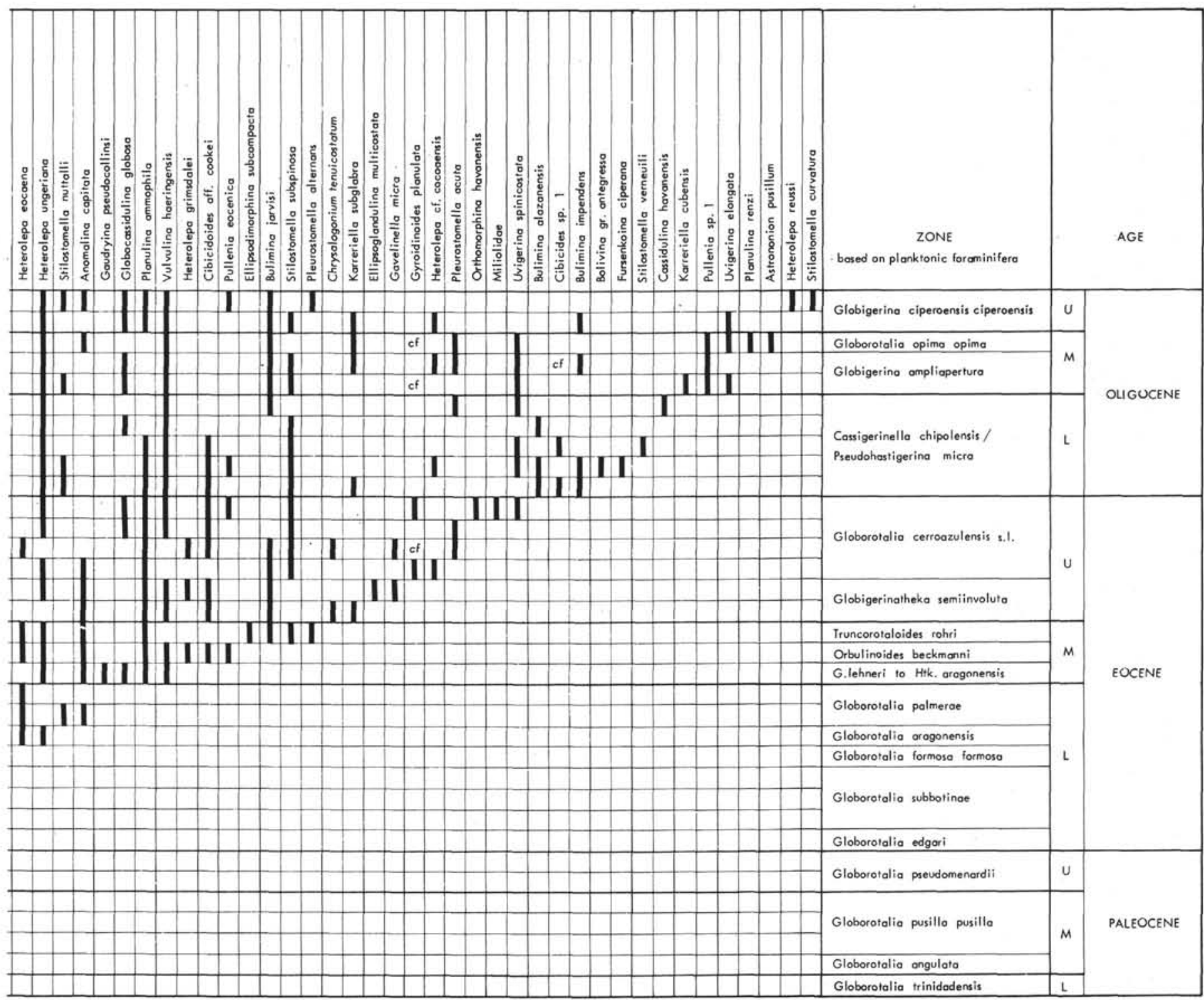

ranges in Sites 360, 361, and 364 from the Paleocene to the upper Eocene. Recent representatives of Stainforthia are recorded from Antarctica and Sweden. The genus thus may be considered to indicate cold to temperate waters.

\section{ANNOTATED SPECIES LIST}

\section{Alabamina dissonata (Cushman and Renz) (Plate 4, Figures 10,11)}

Pulvinulinella atlantisae Cushman, var. dissonata Cushman and Renz, 1948, Cushman Lab. Foram. Res., Spec. Publ. 24, p. 35, pl. 7, fig. 11, 12. Scarce at all sites.

\section{Ammodiscus glabratus Cushman and Jarvis (Plate 1, Figure 1)}

Ammodiscus glabratus Cushman and Jarvis, 1928, Contrib. Cushman Lab. Foram. Res., v. 4, p. 86, pl. 12, fig. 6. Rather common at Site 360 ; rare at Site $362 \mathrm{~A}$.

\section{Anomalina alazanensis spissiformis Cushman and Stainforth} (Plate 5, Figures 12, 13)

Anomalina alazanensis Nuttall, var. spissiformis Cushman and Stainforth, 1945, Cushman Lab. Foram. Res., Spec. Publ. 14, p. 71 , pl. 14, fig. 5. Fairly common at Sites 360, 362A, and 363; rare at Sites 361 and 364 .

\section{Anomalina capitata (Guembel) \\ (Plate 5, Figures 7, 8)}

Rotalia capitata Guembel, 1868, Abh. K. Bayer, Akad. Wiss., II Cl. v. 10, II Abt., p. 653 , pl. 2, fig. 92. Rather scarce at Sites 360, $362 \mathrm{~A}$, and 363 .

\section{Anomalina pompilioides semicribrata Beckmann}

(Plate 5, Figures 5, 6)

Anomalina pompilioides Galloway and Heminway, var. semicribrata Beckmann, 1953, Ecolog. Geol. Helv., v. 46, p. 400, pl. 27, fig. 3, text-fig. 24, 25. Tabulated in the range charts as $A$. pompiliodes. Fairly common at Sites 360 and 363; scarce at Sites $361,362 \mathrm{~A}$, and 364 .

Anomalina? sp. 1

(Plate 5, Figures 14, 15)

Tabulated in the range charts either as Anomalina sp. 1 or Anomalina? sp. This indetermined species is scarce at Sites 360, 361, 363, and 364 .

\section{Aragonia aragonensis (Nuttall)}

(Plate 3, Figure 20)

Textularia aragonensis Nuttall, 1930, J. Paleontol., v. 4, p. 280 , pl. 23, fig. 16. Rare at Sites 360 and 362A. 
TABLE 5

Distribution of Middle Eocene to Lower Paleocene Benthic Foraminifers in Site 364

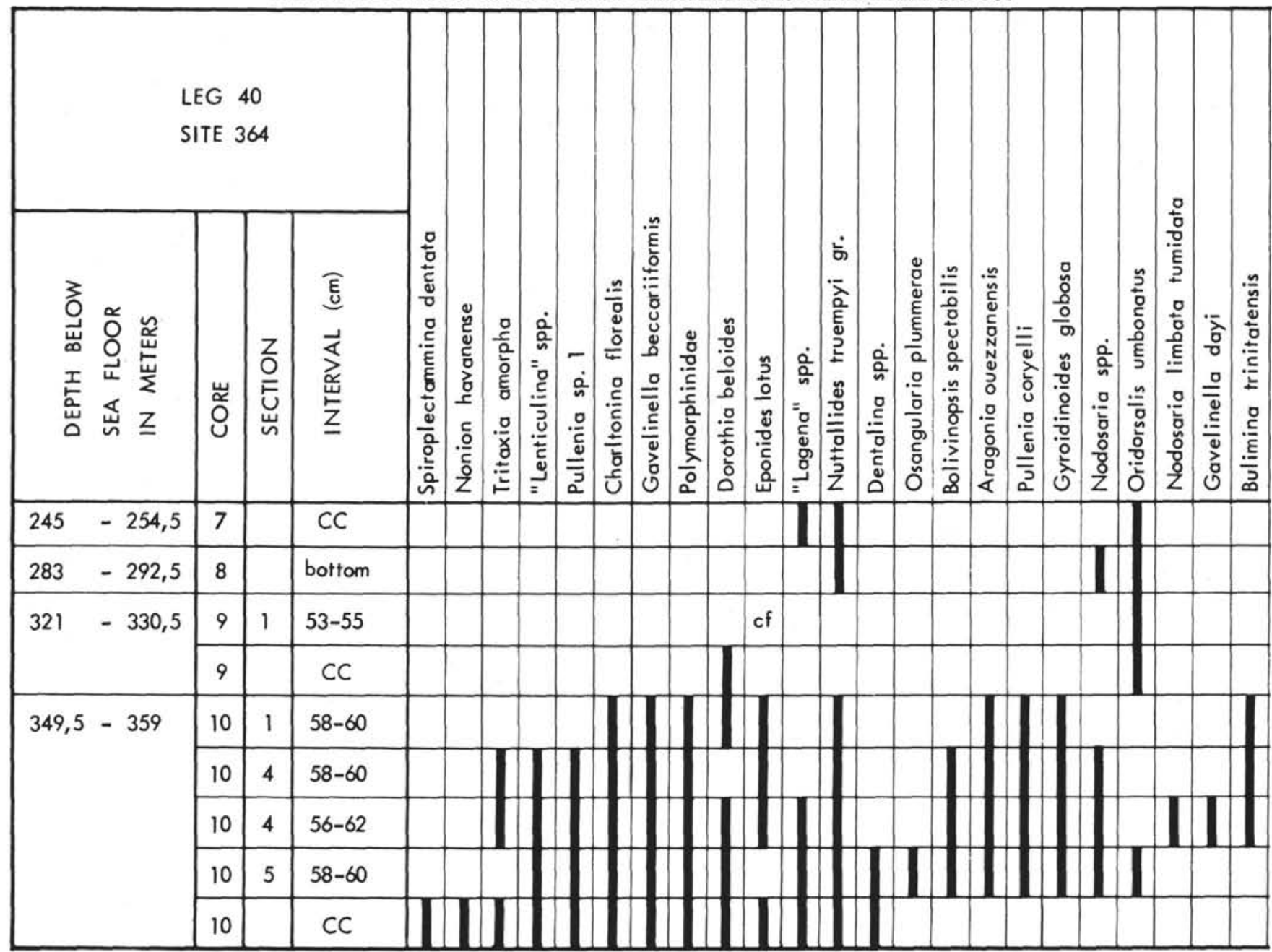

Aragonia ouezzanensis (Rey)

(Plate 3, Figure 22)

Bolivinoides ouezzanensis Rey, 1955, Bull. Soc. Geol. France, 6, v. 4, p. 210, pl. 12, fig. 2. Scarce at Site 364.

\section{Aragonia velascoensis (Cushman) \\ (Plate 3, Figure 21)}

Textularia velascoensis (Cushman) 1925, Contrib. Cushman Lab. Foram. Res., v. 1, p. 18, pl. 3, fig. 1. Rather common at Site 363.

\section{Astrononion pusillum Hornibrook}

Astrononion pussillum Hornibrook, 1961, New Zealand Geol. Surv. Paleontol. Bull., v. 34 (1), p. 96 , pl. 12 , fig. 229,236 . Very rare at Sites 360 and 363.

\section{Bandyella beckmanni Proto Decima and Bolli, n. sp.} (Plate 3, Figure 12)

Virgulina sp. ind. Beckmann, 1953, Ecolog. Geol. Helv., v. 46, p. 367 , pl. 21 , fig. 14,15 .

Test small, short, spindle-shaped. Initial part frequently pointed, rounded in some specimens. Chambers triserially arranged similar to Bulimina, rapidly increasing, inflated and overlapping. Sutures depressed. Aperture subterminal, slightly eccentric, Pleurostomellalike. It differs from the similar Bulimina (Desinobulimina) salisburgensis Hillebrandt, from the Paleocene of Austria, in having a complete triserial arrangement and more inflated chambers.

The new species is placed in Bandyella because this is the only genus that includes a triserial chamber arrangement and a
Pleurostomella-like aperture. It lacks, however, the biserial and uniserial final stage characteristic for Bandyella.

Rare at Sites 360,361, and 364, from the Paleocene to the upper Eocene.

Dimension of holotype: $0.8 \mathrm{~mm}$.

Type locality: Angola Basin, South Atlantic, $11^{\circ} 34^{\prime} \mathrm{S}, 11^{\circ} 58^{\prime} \mathrm{E}$.

Type sample: DSDP Leg 40, Site 364 , Core 10, Section 1, 58-60 $\mathrm{cm}$.

Type stratum: Middle Paleocene, Heliolithus kleinpelli Zone.

Name: The species is named for Jean-Pierre Beckmann, Geological Institute ETH, Zürich.

Depository: Museum of Natural History, Basel, No. C 33881.

\section{Bolivina antegressa Subbotina}

(Plate 2, Figure 2)

Bolivina antegressa Subbotina, 1953, VNIGRI, Trudy, n.s., Sbornik 6, p. 226, pl. 10, fig. 11-16. Scarce at Sites 361, 362A, and 363.

\section{Bolivina striatocarinata Cushman}

Bolivina striatocarinata Cushman, 1936, Cushman Lab. Foram. Res. Spec. Publ., 6, p. 51, pl. 7, fig. 14. Very rare at Site 360.

Bolivinopsis spectabilis (Grzyvowski) (Plate 1, Figure 3)

Spiroplecta spectabilis Grzybowski, 1898, Akad. Univej. Krakowie, Wydr. Mat.-Przyr., Rozpr. 33, p. 293, pl. 12, fig. 12. Rather common at Sites 360, 361, 363, and 364 . 


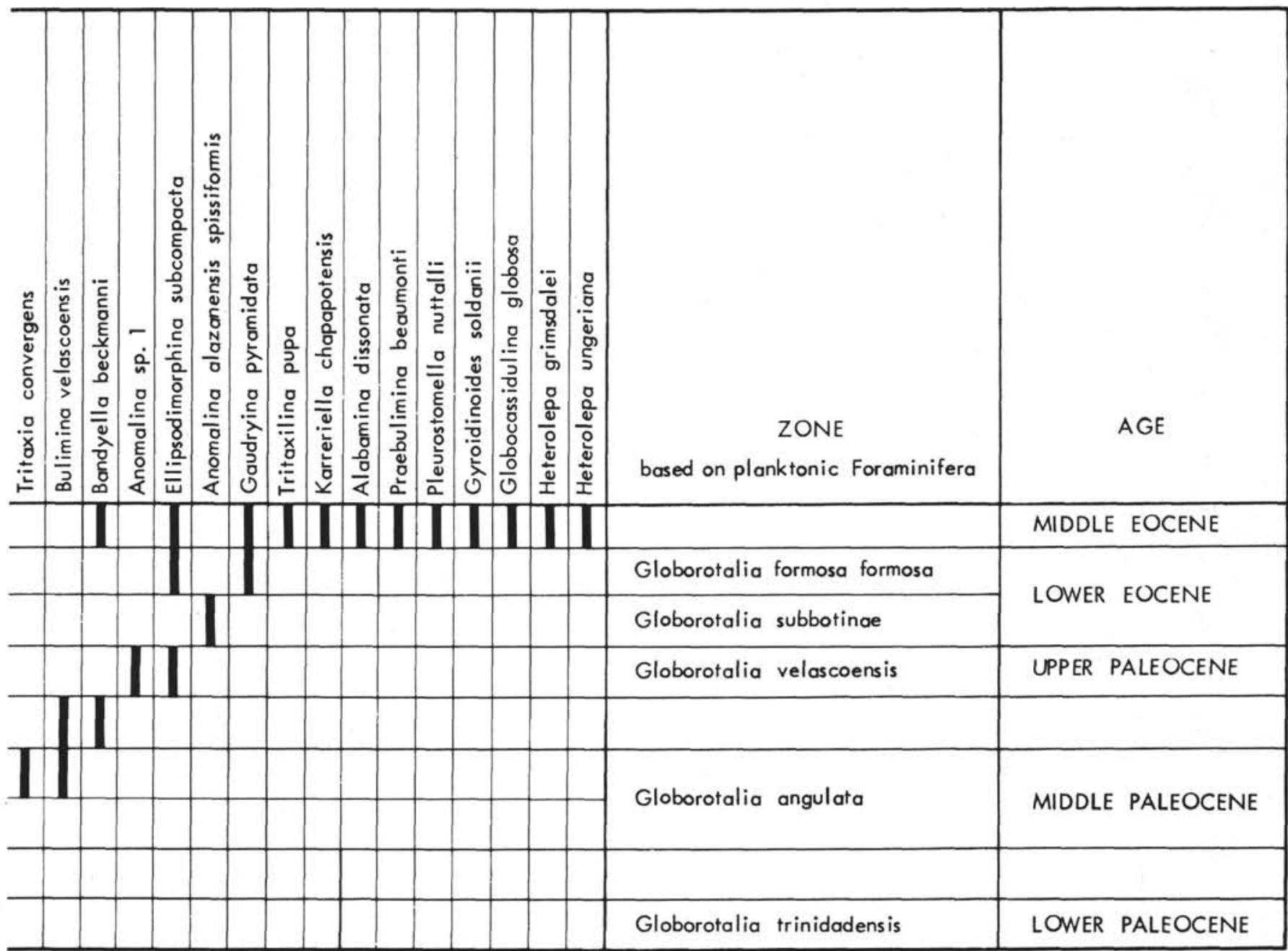

Bulimina alazanensis Cushman (Plate 2, Figure 10)

Bulimina alazanensis Cushman, 1927, J. Paleontol., v. 1, p. 161, pl. 25, fig. 4. Fairly common at Site 363 in the lower Oligocene.

\section{Bulimina alsatica Cushman and Parker}

Bulimina alsatica Cushman and Parker, 1937, Contrib. Cushman Lab. Foram. Res., v. 13, p. 39, pl. 4, fig. 6, 7. Rare at Site 361.

Bulimina impendens Parker and Bermudez (Plate 2, Figures 11, 12)

Bulimina impendens Parker and Bermudez, 1937, J. Paleontol., v. 11, p. 514 , pl. 58 , fig. 7,8 . Rather common at Site 363 .

\section{Bulimina jarvisi Cushman and Parker} (Plate 2, Figure 13)

Bulimina jarvisi Cushman and Parker, 1936, Contrib. Cushman Lab. Foram. Res., v. 12 , p. 29 , pl. 7, fig. 1. Common at Sites 360 , $362 \mathrm{~A}$, and 363 .

\section{Bulimina macilenta Cushman and Parker}

Bulimina macilenta Cushman and Parker, 1947, USGS Prof. Paper 210-D, p. 98, pl. 23, fig. 2, 3. Rare at Site 361.

\section{Bulimina semicostata Nuttall} (Plate 2, Figure 14)

Bulimina semicostata Nuttall, 1930, J. Paleontol., v. 4, p. 285, pl. 23 , fig. 15, 16. Common at Sites 360 and 363, rare at Site 361 .
Bulimina trinitatensis Cushman and Jarvis

(Plate 2, Figures 15, 16)

Bulimina trinitatensis Cushman and Jarvis, 1928, Contrib. Cushman Lab., v. 4 , p. 102 , pl. 14, fig. 12 . Common at Site 363 , rare at Site 364 .

Bulimina tuxpamensis Cole

Bulimina tuxpamensis Cole, 1928, Am. Paleontol. Bull., v. 14, p. 212, pl. 1, fig. 23. Rare at Sites $362 \mathrm{~A}$ and 363.

\section{Bulimina velascoensis (Cushman)}

Gaudryina velascoensis Cushman, 1925, Contrib. Cushman Lab. Foram. Res., v. 1, p. 20, pl. 3, fig. 7. Rare at Site 364.

\section{Cassidulina havanensis Cushman and Bermudez} (Plate 3, Figure 19)

Cassidulina havanensis Cushman and Bermudez, 1936, Contrib. Cushman Lab. Foram. Res., v. 12, p. 36, pl. 6, fig. 11. Rare at Site 363.

\section{Charltonia florealis (White) \\ (Plate 4, Figures 17, 18)}

Gyroidina florealis White, 1928, J. Paleont., v. 2, p. 293, pl. 40, fig. 3. Fairly common at Sites 363 and 364.

\section{Chrysalogonium tenuicostatum Cushman and Bermudez} (Plate 1, Figure 16)

Chrysalogonium tenuicostatum Cushman and Bermudez, 1936, Contrib. Cushman Lab. Foram. Res., v. 12, p. 27, pl. 5, fig. 3-5. Scarce at Sites 360, 361, and 363. 
TABLE 6

Species Present in Cape Basin, Walvis Ridge and Angola Basin

\begin{tabular}{|c|c|c|c|c|c|c|c|c|c|}
\hline & \multicolumn{3}{|c|}{ PALEOCENE } & \multicolumn{3}{|c|}{ EOCENE } & \multicolumn{3}{|c|}{ OLIGOCENE } \\
\hline & L & $M$ & $u$ & L & M & $u$ & L & M & $u$ \\
\hline \multicolumn{10}{|c|}{ Alabamine dissonato } \\
\hline \multicolumn{10}{|c|}{ Anomalino alazanens is spissiformis } \\
\hline \multicolumn{10}{|c|}{ Anomalina sp. 1} \\
\hline \multicolumn{10}{|c|}{ Bolivinopsis spectabilis } \\
\hline \multicolumn{10}{|c|}{ Eponides lotus } \\
\hline \multicolumn{10}{|c|}{ Gavelinello beccariiform is } \\
\hline \multicolumn{10}{|c|}{ Govelinella dayi } \\
\hline \multicolumn{10}{|c|}{ Gyroidinoides globoso } \\
\hline \multicolumn{10}{|c|}{ Gyroidinoides soldanii } \\
\hline Heterolepo grims & & & & $?$ & & & & & \\
\hline \multicolumn{10}{|c|}{ Heterolepo ungeriana } \\
\hline \multicolumn{10}{|c|}{ Nonion hovonense } \\
\hline \multicolumn{10}{|c|}{ Nuttallides truempyi gr. } \\
\hline \multicolumn{10}{|c|}{ Oridorsalis umbonatus gr. } \\
\hline \multicolumn{10}{|c|}{ Pleurostomella nuttalli } \\
\hline \multicolumn{10}{|c|}{ Pullenia coryelli } \\
\hline \multicolumn{10}{|l|}{ Pullenio sp. 1} \\
\hline Tritaxilina pupa & & & & & & & & & \\
\hline
\end{tabular}

TABLE 7

Species Restricted to the Cape Basin

\begin{tabular}{|c|c|c|c|c|c|c|c|c|c|}
\hline & \multicolumn{3}{|c|}{ PALEOCENE } & \multicolumn{3}{|c|}{ EOCENE } & \multicolumn{3}{|c|}{ OLIGOCENE } \\
\hline & L & M & $u$ & L & M & u & L & M & u \\
\hline \multicolumn{10}{|l|}{ Bolivina striatocarinata } \\
\hline \multicolumn{10}{|l|}{ Bulimina alsatica } \\
\hline \multicolumn{10}{|l|}{ Bulimina macilenta } \\
\hline \multicolumn{10}{|l|}{ Clavulina aff. anglica } \\
\hline \multicolumn{10}{|l|}{ Dorothio biformis } \\
\hline \multicolumn{10}{|l|}{ Karreriella boceato } \\
\hline \multicolumn{10}{|l|}{ Karreriella hantkeniana } \\
\hline \multicolumn{10}{|l|}{ Loxostomoides dupuyi } \\
\hline \multicolumn{10}{|l|}{ Nodosaria longiscata } \\
\hline \multicolumn{10}{|l|}{ Nodosaria sp. 1} \\
\hline \multicolumn{10}{|l|}{ Proebulimino grata } \\
\hline \multicolumn{10}{|l|}{ Rectuvigerina elegans } \\
\hline \multicolumn{10}{|l|}{ Siphotextularia finlayi } \\
\hline Stainforthia ryani & $?$ & & & $?$ & & & & & \\
\hline \multicolumn{10}{|c|}{ Stilostomella consobrina } \\
\hline \multicolumn{10}{|c|}{ Stilostomella nutralli aculeata } \\
\hline \multicolumn{10}{|c|}{ Stilostomella ef. stachei } \\
\hline \multicolumn{10}{|l|}{ Tappanino selmensis } \\
\hline \multicolumn{10}{|l|}{ Uvigerina chirana } \\
\hline \multicolumn{10}{|l|}{ Uvigerina ef. eocaena } \\
\hline Uvigerina gallowoyi & & & & & & & & & \\
\hline
\end{tabular}

\section{Cibicidoides alleni (Plummer)}

(Plate 5, Figures 18, 19)

Truncatulina alleni Plummer, 1926, Texas Univ. Bull. 2644, p. 144, pl. 10, fig. 4. Scarce at Site 363.

\section{Cibicidoides aff. cookei (Cushman and Garrett)}

(Plate 5, Figures 16, 17)

Cibicides cookei Cushman and Garrett, 1938, Contrib. Cushman Lab. Foram. Res., v. 14, p. 65, pl. 11, fig. 3. Rather common at Sites 360 and 363, scarce at Sites 361 and 362A.

\section{Clavulina aff. anglica (Cushman)}

(Plate 1, Figure 11)

Pseudoclavulina anglica Cushman, 1936, Cushman Lab. Foram. Res., Spec. Publ., 6, p. 18, pl. 3, fig. 5. Scarce at Site 360, tabulated in the range chart as Clavulina $\mathrm{sp}$.

\section{Clavulina cocoaensis (Cushman)}

(Plate 1, Figure 12)

Pseudoclavulina cocoaensis Cushman, 1936, Cushman Lab. Foram. Res., Spec. Publ., 6, p. 18, pl. 3, fig. 6. Rare at Site 362A.
TABLE 8

Species Restricted to the Walvis Ridge

\begin{tabular}{|c|c|c|c|c|c|c|c|c|c|}
\hline & \multicolumn{3}{|c|}{ PALEOCENE } & \multicolumn{3}{|c|}{ EOCENE } & \multicolumn{3}{|c|}{ OLIGOCENE } \\
\hline & L & M & $u$ & $\mathrm{t}$ & M & $u$ & $\mathrm{~L}$ & M & $u$ \\
\hline \multicolumn{10}{|l|}{ Aragonia veloscoens is } \\
\hline \multicolumn{10}{|l|}{ Bulimina alazanens is } \\
\hline \multicolumn{10}{|l|}{ Bulimina impendens } \\
\hline \multicolumn{10}{|l|}{ Bulimina fuxpamensis } \\
\hline \multicolumn{10}{|l|}{ Cassidulina havanens is } \\
\hline \multicolumn{10}{|l|}{ Cibicidoides alleni } \\
\hline \multicolumn{10}{|c|}{ Clavulina cocosensis } \\
\hline \multicolumn{10}{|c|}{ Coryphostoma ef. limonense } \\
\hline \multicolumn{10}{|c|}{ Goudryina pyramidata } \\
\hline \multicolumn{10}{|l|}{ Gavelinella rubiginosa } \\
\hline \multicolumn{10}{|l|}{ Globocassidulino oblongo } \\
\hline \multicolumn{10}{|l|}{ Gyroidinoides subangulato } \\
\hline \multicolumn{10}{|c|}{ Heterolepo cf. cocooensis } \\
\hline \multicolumn{10}{|l|}{ Marssonella nocatoensis } \\
\hline \multicolumn{10}{|l|}{ Marssonella traubi } \\
\hline \multicolumn{10}{|l|}{ Neoflabellina jarvisi } \\
\hline \multicolumn{10}{|c|}{ Neoflabellina semireticulato } \\
\hline \multicolumn{10}{|c|}{ Orthomorphino havanensis } \\
\hline \multicolumn{10}{|l|}{ Orthomorphina rohri } \\
\hline \multicolumn{10}{|l|}{ Plectina dalmatina } \\
\hline \multicolumn{10}{|l|}{ Pleurostomella obesa } \\
\hline \multicolumn{10}{|l|}{ Praebulimina beoumonti } \\
\hline \multicolumn{10}{|l|}{ Pullenia jarvisi } \\
\hline \multicolumn{10}{|l|}{ Remesella varians } \\
\hline Spiroplectammina excola & & & & & & & & & \\
\hline Stilostomella curvatura & & & & & & & & & \\
\hline Tritaxia ospera & & - & & & & & $\cdots$ & & \\
\hline Tritaxia trilatero & & & & & & & $\because 1$ & & 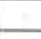 \\
\hline Tritaxilino cubensis & & & & & & & & & \\
\hline Uvigerina sp. & & & & & & & & & \\
\hline Uvigerina biserialis & & & & & & & & & \\
\hline Uvigerina oculocostata & & & & & & & 2 & & \\
\hline
\end{tabular}

TABLE 9

Species Restricted to the Angola Basin

\begin{tabular}{|c|c|c|c|c|c|c|c|c|c|}
\hline & \multicolumn{3}{|c|}{ PALEOCENE } & \multicolumn{3}{|c|}{ EOCENE } & \multicolumn{3}{|c|}{ OLIGOCENE } \\
\hline & L & M & $u$ & L & M & $u$ & $\mathrm{~L}$ & M & u \\
\hline Arogonio ovezzonensis & & & & & & & & & \\
\hline Bulimina veloscoensis & & & & & & & & & \\
\hline Osongularia plummerae & & & & & & & & & \\
\hline Tritoxio amorpha & & & & & & & & & \\
\hline
\end{tabular}

Coryphostoma ef. limonense (Cushman)

(Plate 2, Figure 1)

cf. Bolivina incrassata Reuss, var. limonensis Cushman, 1926, Contrib. Cushman Lab. Foram. Res., v. 2, p. 19, pl. 2, fig. 2. Scarce at Site 363.

\section{Dorothia beloides Hillebrandt}

(Plate 1, Figure 10)

Dorothia beloides Hillebrandt, 1962, Bayer. Ak. Wiss. Mat.-Nat. K1. Abh. N.F., Heft 108, p. 39, pl. 2, fig. 8-14; pl. 15, fig. 12,13, text-fig. 3. Rather scarce at Sites 360 and 364.

\section{Dorothia biformis Finlay}

Dorothia biformis Finlay, 1939, Roy. Soc. New Zealand Trans. Proc., v. 69 , p. 313 , pl. 25 , fig. $26-28$. Rare at Site 360.

\section{Dorothia brevis Cushman and Stainforth}

Dorothia brevis Cushman and Stainforth, 1945, Cushman Lab. Foram. Res., Spec. Publ. 14, p. 18, pl. 2, fig. 5. Rare at Sites 360, 361 , and 363 . 
TABLE 10

Species Present in the Paleocene of the Cape Basin, Walvis Ridge, and Angola Basin ${ }^{\mathrm{a}}$

\begin{tabular}{|c|c|c|c|c|c|c|c|c|c|c|}
\hline & & CAPE BASIN & WALVIS RIDGE & ANGOUA BASIN & & THER & $R$ LO & pais & LITIE & \\
\hline & & Site 361 & Site 363 & Site 364 & c & L & M & $p$ & R & $\mathrm{v}$ \\
\hline Arogonio ovezzonensis & * & 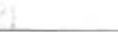 & & & & & & & - & \\
\hline Arogonio velascoencis & * & 2 & & & & | & & - & - & - \\
\hline Bulimina trinitotonsis & & & & & & $\bullet$ & & - & $\bullet$ & \\
\hline Bulimina veloncoenais & & F. & & & - & & & & - & - \\
\hline Cheritonine flereolis & * & 3 & & & & $\bullet$ & & & - & - \\
\hline Cibicidoides olleni & & ti & & & & & - & - & $\bullet$ & \\
\hline Corpphostomo of. limonense & * & 4 & & & & $\bullet$ & & - & $\bullet$ & \\
\hline Dentaline spp. & & & & & & & & & & \\
\hline Dorothio beloides & + & & & & & & & & $\bullet$ & \\
\hline Dorothia brevis & + & & & & & & & & & \\
\hline Eponides lotur & + & & & & - & - & & - & $\bullet$ & - \\
\hline Goudryine pyromidato & $*$ & - & & & & $\bullet$ & & & $\bullet$ & $\bullet$ \\
\hline Gavelinello beccorififormis & * & & & & - & $\bullet$ & & - & - & $\cdot$ \\
\hline Gavelinella dayi & & & & & & $\bullet$ & & & - & - \\
\hline Govelinello rubiginose & + & & & & & & & & & \\
\hline Govelinello veloscoentin & * & $=$ & & & & T. & & - & $\bullet$ & • \\
\hline Gyroidinoiden globone & * & - & & & & $\bullet$ & & & $\bullet$ & \\
\hline Logene upp. & & 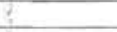 & & & & & & & & \\
\hline Lenticuline spp. & & $i$ & & & & 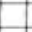 & & & 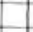 & \\
\hline Mortonello nocatoentis & & & & & & - & & & - & - \\
\hline Neoflabellina jervisi & & 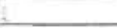 & & 1 & $\bullet$ & $\bullet$ & & $\bullet$ & - & \\
\hline Nooflabelline semireticulato & & 1 & & & & - & & $\bullet$ & $\bullet$ & \\
\hline Nodostoria upp. & & & & & & & & & & \\
\hline Nuttallider truempyi & + & & & & $\bullet$ & - & & $\bullet$ & $\bullet$ & \\
\hline Orangulario plummeroe & & & & & & & - & & $\bullet$ & \\
\hline Polymorphinidae & & 3 & & & & & & & & \\
\hline Proebulimine beoumonti & $*$ & & & & & $\bullet$ & & & - & \\
\hline Pullenic coryellt & * & & & & & - & & - & - & - \\
\hline Pullenia jorvigi & & & & & & $\bullet$ & & - & $\bullet$ & \\
\hline Pullenio sp. 1 & + & & & & & & & . & ( & \\
\hline Remesella varions & & & & & - & $\bullet$ & & - & $\bullet$ & \\
\hline Stoinforthia ryoni & + & $?$ & & & & & & & & \\
\hline Spiroplectommina dentato & * & & & & $\bullet$ & - & & $\bullet$ & $\bullet$ & \\
\hline Spiroplectommina exeolato & & & & & & $\bullet$ & & $\bullet$ & $\bullet$ & $\bullet$ \\
\hline Tritoxio omorpho & & & & & & $\bullet$ & & $\bullet$ & & - \\
\hline Tritexio oupero & & & & & & $\bullet$ & & $\bullet$ & & - \\
\hline Tritoxia trilatero & * & & & & & $\bullet$ & & $\bullet$ & & $\bullet$ \\
\hline Tritaxiling cubersin & & & & & & & & $\bullet$ & & \\
\hline
\end{tabular}

${ }^{a}$ The letters in the column to the right indicates that the specie is present also in the Caucasus (C), Lizard Springs Foramtion of Trinidad (L), Midway Formation (M), Passagno Section in Italy (P) Reichenhall and Salzburg basins in Austria (R), and in Velasco Formation (V). An asterisk indicates that the species is also present in the Upper Cretaceous of Leg 40, a cross that the species continues into the Eocene of Leg 40.

Ellipsodimorphina subcompacta Liebus (Plate 3, Figure 14)

Ellipsodimorphina subCompacta Liebus, 1922, Lotos (Prag), v. 70, p. 57, pl. 2, fig. 13. Scarce at Sites 360, 362A, 363, and 364.

Ellipsoglandulina labiata (Schwager)

Glandulina labiata Schwager, 1866, Novara-Exped., Geol. Theîl, v. 2, p. 237 , pl. 6 , fig. 77 . Scarce at Sites 360 and $362 \mathrm{~A}$.

Ellipsoglandulina multicostata (Galloway and Morrey) (Plate 3, Figures 16, 17)

Daucina multicostata Galloway and Morrey, 1929, Am. Paleontol. Bull., v. 15, 55, p. 42, pl. 6, fig. 13. Rare at Sites 361 and 363.

\section{Ellipsoidina ellipsoides Seguenza}

(Plate 3, Figure 15)

Ellipsoidina ellipsoides Seguenza, 1859, Eco Peloritano, Messina, ser. 2, anno 5, fasc. 9, pl. 12, fig. 1-3. Scarce at Site 360, rare at Site $362 \mathrm{~A}$.

\section{Eponides lotus (Schwager)}

(Plate 3, Figures 3, 4)

Pulvinulina lotus Schwager, 1883, Paleontogr., v. 30, Abh. 1. Scarce at Sites 360, 363, and 364.
TABLE 11

Species Restricted to the Eocene of Leg 40

\begin{tabular}{|c|c|c|c|c|c|}
\hline & \multicolumn{2}{|c|}{ CAPE BASIN } & \multicolumn{2}{|c|}{ WALVIS RIDGE } & \multirow{2}{*}{$\begin{array}{c}\text { ANGOLA BASIN } \\
\text { Site } 364\end{array}$} \\
\hline & Site 360 & Site 361 & Site 362A & Site 363 & \\
\hline \multicolumn{6}{|l|}{ Ammodiscus glabratus } \\
\hline \multicolumn{6}{|l|}{ Arogonia arogonensis } \\
\hline \multicolumn{6}{|l|}{ Bulimina alsatica } \\
\hline \multicolumn{6}{|c|}{ Bulimina macilenta } \\
\hline \multicolumn{6}{|c|}{ Chrysalogonium tenuicostatum } \\
\hline \multicolumn{6}{|l|}{ Clavulina cocoaensis } \\
\hline \multicolumn{6}{|l|}{ Dorothia biformis } \\
\hline \multicolumn{6}{|c|}{ Ellipsodimorphina subcompacto } \\
\hline \multicolumn{6}{|c|}{ Ellipsoglandulino multicostato } \\
\hline \multicolumn{6}{|c|}{ Glomospiro charoides } \\
\hline \multicolumn{6}{|l|}{ Karreriella chapapotensis } \\
\hline \multicolumn{6}{|l|}{ Loxostomoides dupuyi } \\
\hline \multicolumn{6}{|l|}{ Nodosaria longiscata } \\
\hline \multicolumn{6}{|l|}{ Nodosaria sp. 1} \\
\hline \multicolumn{6}{|c|}{ Orthomorphina havanensis } \\
\hline \multicolumn{6}{|l|}{ Plectina dalmatina } \\
\hline \multicolumn{6}{|l|}{ Pleurostomella obesa } \\
\hline \multicolumn{6}{|l|}{ Rectuvigerina elegans } \\
\hline \multicolumn{6}{|l|}{ Siphotextulario finlayi } \\
\hline \multicolumn{6}{|l|}{ Stilostomella consobrina } \\
\hline \multicolumn{6}{|c|}{ Stilostomella nuttalli aculeata } \\
\hline \multicolumn{6}{|l|}{ Stilostomella ef. stachei } \\
\hline \multicolumn{6}{|l|}{ Tappanina selmensis } \\
\hline \multicolumn{6}{|l|}{ Uvigerina chirana } \\
\hline \multicolumn{6}{|l|}{ Uvigerina eocaena } \\
\hline \multicolumn{6}{|l|}{ Uvigerina biserialis } \\
\hline Uvigerina acutocostato & & & & & \\
\hline
\end{tabular}

Fursenkoina ciperana (Cushman and Stainforth)

Virgulina ciperana Cushman and Stainforth, 1945, Cushman Lab. Foram. Res., Spec, Publ., 14, p. 46, pl. 7, fig. 10. Rare at Sites 360 and 363.

Gaudryina pseudocollinsi Cushman and Stainforth (Plate 1, Figure 7)

Gaudryina pseudocollinsi Cushman and Stainforth, 1945, Cushman Lab. Foram. Res., Spec. Publ., 14, p. 17, pl. 2, fig. 1-3. Rare at Sites 360, 362A, and 363 .

\section{Gaudryina pyramidata Cushman}

(Plate 1, Figure 6)

Gaudryina laevigata Franke, var. pyramidata Cushman, 1926, Am. Assoc. Petrol. Geol. Bull., v. 10, p. 587, pl. 16, fig. 8. Rare at Site 363.

\section{Gavelinella beccariiformis (White)}

(Plate 6, Figures 3, 4)

Rotalia beccariiformis White, 1928, J. Paleontol., v. 2, p. 287, pl. 39, fig. 2-4. Rare at Site 361, common at Sites 363 and 364 .

\section{Gavelinella dayi (White)}

(Plate 6, Figures 1, 2)

Planulina dayi White, 1928, J. Paleontol., v. 2, p. 300 , pl. 41, fig. 3 . Rare at Sites 361 and 364, rather common at Site 363.

Gavelinella micra (Bermudez)

(Plate 6, Figures 5, 6)

Cibicides micrus Bermudez, 1949, Cushman Lab. Foram. Res., Spec. Publ., 25, p. 302 , pl. 24, fig. $34-36$. Rather common at Sites 360 and $362 \mathrm{~A}$.

\section{Gavelinella rubiginosa (Cushman)}

(Plate 6, Figures 7,8 )

Anomalina rubiginosa Cushman, 1926, Am. Assoc. Petrol. Geol. Bull., v. 10, p. 607, pl. 21, fig. 6. Rather common at Site 363. 
TABLE 12

Species Restricted to the Oligocene of Leg 40

\begin{tabular}{|c|c|c|c|}
\hline & \multirow{2}{*}{$\begin{array}{c}\text { CAPE BASIN } \\
\text { Site } 360\end{array}$} & \multicolumn{2}{|c|}{ WALVIS RIDGE } \\
\hline & & Site $362 \mathrm{~A}$ & Site 363 \\
\hline \multicolumn{4}{|l|}{ Astrononion pusillum } \\
\hline \multicolumn{4}{|c|}{ Bolivina striatocarinata } \\
\hline \multicolumn{4}{|l|}{ Bulimina alazonensis } \\
\hline \multicolumn{4}{|l|}{ Bulimina impendens } \\
\hline \multicolumn{4}{|l|}{ Cossidulina havanersis } \\
\hline \multicolumn{4}{|c|}{ Cibicides sp. 1} \\
\hline \multicolumn{4}{|c|}{ Globocassidulina oblonga } \\
\hline \multicolumn{4}{|l|}{ Heterol epa reussi } \\
\hline \multicolumn{4}{|l|}{ Karreriella boccato } \\
\hline \multicolumn{4}{|c|}{ Karreriella hantkeniana } \\
\hline \multicolumn{4}{|c|}{ Planulino renzi } \\
\hline \multicolumn{4}{|l|}{ Stilostomella curvatura } \\
\hline \multicolumn{4}{|l|}{ Uvigerina sp. } \\
\hline Uvigerina gallowayi & & & \\
\hline
\end{tabular}

Gavelinella velascoensis (Cushman)

Anomalina velascoensis Cushman, 1925, Contrib. Cushman Lab. Foram. Res., v. 1, p. 21, pl. 3, fig. 3. Rare at Sites 361 and 363.

\section{Globocassidulina globosa (Hantken)}

(Plate 3, Figures 23, 24)

Cassidulina globosa Hantken, 1875, Ungar. Geol. Anst., Mitt. Jb., v. 4 , p. 64 , pl. 16, fig. 2 . Rather common at Sites 360,361 , $362 \mathrm{~A}$, and 363 .

\section{Globocassidulina oblonga (Reuss)}

Cassidulina oblonga Reuss, 1850, K. Akad. Wiss., Math.-Nat. Cl., Denkschr., v. 1, p. 376, pl. 48 , fig. 5,6 .

\section{Glomospira charoides (Jones and Parker)} (Plate 1, Figure 2)

Trochammina squamata Jones and Parker, var. charoides Jones and Parker, 1860, Quart. J. Geol. Soc. London, v. 16, p. 304. Scarce at Sites 360 and $362 \mathrm{~A}$.

\section{Gyroidinoides globosa (Hagenow)}

(Plate 4, Figures 19, 20)

Nonionina globosa Hagenow, 1842, N. Jb. Min., p. 574. Rather common at Sites 361, 363, and 364 .

\section{Gyroidinoides octocamerata (Cushman and Hanna)} (Plate 5, Figures 3, 4)

Gyroidina soldanii d'Orbigny, var. octocamerata Cushman and Hanna, 1927, Calif. Acad. Sci. Proc., S. 4, v. 16, p. 223, pl. 14 , fig. 16-18. Rather common at Site 360 .

\section{Gyroidinoides planulata (Cushman and Renz)} (Plate 4, Figures 13, 14)

Gyroidina planulata Cushman and Renz, 1941, Contrib. Cushman Lab. Foram. Res., v. 17, p. 23, pl. 4, fig. 1. Rather common at Sites $360,362 \mathrm{~A}$, and 363 .

\section{Gyroidinoides soldanii (d'Orbigny)}

$$
\text { (Plate 5, Figures 1, 2) }
$$

Rotalina soldanii d'Orbigny, 1826, Ann. Sc. Nat. s. 1, v. 7, p. 278. Common at all sites.

\section{Gyroidinoides subangulata (Plummer)}

Rotalia soldanii (d'Orbigny) var. subangulata Plummer, 1926, Univ. Texas Bull., 2644, p. 154, pl. 12, fig. 1. Scarce at Sites 362A and 363

\section{Heterolepa cf. cocoaensis (Cushman)}

cf. Eponides cocoaensis Cushman, 1928, Contrib. Cushman Lab. Foram. Res., v. 4, p. 73, pl. 10, fig. 2. Scarce at Site 363.
Heterolepa eocaena (Guembel)

(Plate 6, Figures 14, 15)

Rotalia eocaena Guembel, 1868, Abh. K. Bayer. Akad. Wiss., II cl., v. 10, Il Abt., p. 650, pl. 2, fig. 87. Rare at Sites 360 and 361; common at Sites $362 \mathrm{~A}$ and 363 .

\section{Heterolepa grimsdalei (Nuttall)}

(Plate 6, Figures 16, 17)

Cibicides grimsdalei Nuttall, 1930, J. Paleontol., v. 4, p. 291, pl. 25 , fig. 7, 8, 11. Common at Site 360; scarce at Sites 361, 362A, 363, and 364 .

\section{Heterolepa pygmea (Hantken)}

(Plate 6, Figure 13)

Pulvinulina pygmea Hantken, 1875, Ungar. Geol. Anst. Mitt. Jb., v. 4 , n. 1 , p. 78 , pl. 10 , fig. 8 . Scarce at Sites $360,361,362$ A.

\section{Heterolepa reussi (Silvestri}

(Plate 6, Figures 9-12)

Truncatulina dutemplei d’Orbigny, var. reussi Silvestri, 1906, Riv. 5 , Paleontol., v. 12, p. 33. Rare at Sites 360, 362A, and 363.

\section{Heterolepa ungeriana (d'Orbigny)}

(Plate 6, Figures 18, 19)

Rotalina ungeriana d'Orbigny, 1864, Foram. Bassin Tert. Vienne, p. 157 , pl. 8 , fig. 16-18. Common at all sites.

\section{Karreriella baccata (Schwager)}

Gaudryina baccata Schwager, 1866, Novara Exped., Geol. Theil., v. 2 , p. 200 , pl. 4 , fig. 12 . Scarce at Site 360 .

\section{Karreriella chapapotensis (Cole)}

Textularia chapapotensis Cole, 1928, Am. Paleontol. Bull., v. 14, n. 53, p. 6, pl. 2, fig. 9. Scarce at Sites 360,361 , and 364 .

\section{Karreriella cubensis Cushman and Bermudez}

Karreriella cubensis Cushman and Bermudez, 1937, Contrib. Cushman Lab. Foram. Res., v. 13, p. 4, pl. 1, fig. 18, 19. Scarce at Sites 360,361 , and 363 .

\section{Karreriella hantkeniana Cushman}

Karreriella hantkeniana Cushman, 1936, Cushman Lab. Foram. Res., Spec. Publ., 6, p. 36, pl. 5, fig. 19. Rare at Site 360.

\section{Karreriella siphonella (Reuss)}

Gaudryina siphonella Reuss, 1851, Z. Deut. Geol. Ges., v. 3, p. 78, pl. 5, fig. 40-42. Rare at Sites 360 and 362A.

\section{Karreriella subglabra (Guembel)} (Plate 1, Figure 9)

Gaudryina subglara Guembel, 1868, Abh. K. Bayer Akad. Wiss., II cl., v. 10 , II. Abt. p. 602 , pl. 1, fig. 4. Rather common at Sites $360,362 \mathrm{~A}$, and 363 .

\section{Laticarinina of, bullbrooki Cushman and Todd}

cf. Laticarinina bullbrooki Cushman and Todd, 1942, Contrib. Cushman Lab. Foram. Res., v. 18, p. 19, pl. 4, fig. 8, 9. Very rare at Site 360 .

\section{Loxostomoides dupuyi (Colom)}

(Plate 2, Figures 3, 4)

Rectobolivina dupuyi Colom, 1954, Inst. Geol. Min. España, Bol., v. 66, p. 181, pl. 7, fig. 5-10. Rare at Site 361.

\section{Marssonella nacataensis (White)}

(Plate 1, Figure 15)

Textularia nacataensis White, 1929, J. Paleontol., v. 2, p. 31, pl. 4, fig. 2. Scarce at Site 363. 
Marssonella traubi Hagn.

Marssonella traubi Hagn, 1956, Palaeontographica, Abt. A, v. 107, p. 118 , pl. 9, fig. 20, text-fig. 5, 6. Scarce at Site $362 \mathrm{~A}$

\section{Neoflabellina jarvisi (Cushman)}

Flabellina jarvisi Cushman, 1935, Contrib. Cushman Lab. Foram. Res., v. 11, p. 85 , pl. 13, fig. 7,8 . Rare at Site 363 .

\section{Neoflabellina semireticulata (Cushman and Jarvis)} (Plate 1, Figure 18)

Flabellina semireticulata Cushman and Jarvis, 1928, Contrib. Cushman Lab. Foram. Res., v. 4, p. 98, pl. 13, fig. 14. Scarce at Site 363.

\section{Nodosarella subnodosa (Guppy)}

(Plate 3, Figure 18)

Ellipsoidina subnodosa Guppy, 1894, Proc. Zod. Soc. London, p. 650 , pl. 41, fig. 12. Rare at Sites 360 and 362A.

\section{Nodosaria longiscata d'Orbigny}

Nodosaria longiscata d'Orbigny, 1846, Foram. Bassin Tert. Vienne, p. 32, pl. 7, fig. 10-12. Rather common at Sites 360 and 361.

\section{Nonion havanense Cushman and Bermudez (Plate 4, Figures 1, 2)}

Nonion havanense Cushman and Bermudez, 1937, Contrib. Cushman Lab. Foram. Res., v. 13, p. 19, pl. 2, fig. 13, 14. Rather common at all sites.

\section{Nutallides truempyi (Nuttall)}

(Plate 3, Figures 1, 2)

Eponides truempyi Nuttall, 1930, J. Paleontol., v. 4, p. 287, pl. 24 , fig. $9,13,14$. Common at all sites

\section{Oridorsalis umbonatus (Reuss)}

Rotalina umbonata Reuss, 1851, Z. Deut. Geol. Ges., v. 3, p. 75 , pl. 5, fig. 35. Common at all sites.

\section{Orthomorphina havanensis (Cushman and Bermudez)}

Nodogenerina havanensis Cushman and Bermudez, 1937, Contrib. Cushman Lab. Foram. Res., v. 13, p. 14, pl. 1, fig. 47, 48.

\section{Orthomorphina rohri (Cushman and Stainforth)} (Plate 1, Figure 17)

Nodogenerina rohri Cushman and Stainforth, 1945, Cushman Lab. Foram. Res., Spec, Publ., 14, p. 39, pl. 5, fig. 26. Rare at Site 363.

\section{Osangularia pteromphalia (Guembel)}

(Plate 4, Figures 15, 16)

Rotalia pteromphalia Guembel, 1868, Abh. K. Bayer. Akad. Wiss., Il cl. v. 10, II Abt. p. 651, pl. 2, fig. 88. Rather common at Sites $360,362 \mathrm{~A}$, and 363 .

\section{Osangularia plummerae Brotzen}

Osangularia plummerae Brotzen, 1940, Sver. Geol. Unders., Avh., s.c., n. 435 , p. 30 , pl. 10, fig. 1, 2. Rare at Sites 364.

\section{Planulina ammophila (Guembel)}

(Plate 3, Figures 5, 6)

Rotalia ammophila Guembel, 1868, Abh, k. Bayer. Akad. Wiss., II Cl., v. 10, II Abt., p. 652, pl. 2. Rather common at Sites 360, 361 and $362 \mathrm{~A}$.

\section{Planulina renzi Cushman and Stainforth} (Plate 3, Figure7)

Planulina renzi Cushman and Stainforth, 1945, Cushman Lab. Foram. Res., Spec. Publ., 14, p. 72, pl. 15, fig. 1. Rare at Sites 360 and 363 .

\section{Plectina dalmatina (Schubert)}

(Plate 1, Figure 13)

Gaudryina dalmatina Schubert, 1911, in Liebus, Sitz. Akad. Wiss. Wien, v. 120 , p. 75 , pl. 3, fig. 5, text-fig. 4 . Scarce at Sites 362A and 363 .

\section{Pleurostomella acuta Hantken}

(Plate 3, Figure 9)

Pleurostomella acuta Hantken, 1875, Ungar. Geol. Anst., Mitt. Jb., v. 4 , n. 1, p. 44 , pl. 13, fig. 18. Rather common at Sites 360 , $362 \mathrm{~A}$, and 363 .

\section{Pleurostomella alternans Schwager} (Plate 3, Figure 10)

Pleurostomella alternans Schwager, 1966, Novara Exped., Geol. Theil., v. 2, p. 238, pl. 6, fig. 79-80. Rare at Sites 360 and 363.

Pleurostomella incrassata Hantken (Plate 3, Figure 11)

Pleutostomella incrassata Hantken, 1884, Math. u. naturw. Ber. Ung. v. 2, p. 146, pl. 1, fig. 4,7 . Rather common at Sites 360,361 , and $362 \mathrm{~A}$.

\section{Pleurostomella nuttalli Cushman and Siegfus} (Plate 3, Figure 13)

Pleurostomella nuttalli Cushman and Siegfus, 1939, Contrib. Cushman Lab. Foram. Res., v. 15, p. 29, pl. 6, fig. 17, 18 Rather common at all sites.

\section{Pleurostomella obesa Cushman and Bermudez}

Pleurostomella obesa Cushman and Bermudez, 1937, Contrib. Cushman Lab. Foram. Res., v. 13, p. 16, pl. 1, fig. 61. Rare at Site $362 \mathrm{~A}$.

\section{Praebulimina beaumonti (Cushman and Renz) (Plate 1, Figure 19)}

Buliminella beaumonti Cushman and Renz, 1946, Cushman Lab. Foram. Res., Spec. Publ., 18, p. 36, pl. 6, fig. 7. Scarce at Sites 363 and 364 .

\section{Praebulimina grata (Parker and Bermudez) (Plate 1, Figure 20)}

Bulimenella grata Parker and Bermudez, 1937, J. Paleontol., v. 11, p. 515 , pl. 59, fig. 6 . Scarce at Sites 360 and 361 .

\section{Pullenia coryelli White \\ (Plate 4, Figures 3, 4)}

Pullenia coryelli White, 1929, J. Paleontol. v. 3, p. 56, pl. 5, fig. 22. Scarce at Sites 361, 363, and 364.

\section{Pullenia eocenica Cushman and Siegfus}

(Plate 4, Figures 7, 8)

Pullenia eocenica Cushman and Siegfus, 1939, Contrib. Cushman Lab. Foram. Res., v. 15, p. 31, pl. 7, fig. 1. Rather common at Sites $360,316,362 \mathrm{~A}$, and 363 .

\section{Pullenia jarvisi Cushman}

Pullenia jarvisi Cushman, 1936, Contrib. Cushman Lab. Foram. Res. v. 12 , p. 77 , pl. 13, fig. 6 . Rare at Site 363.

\section{Pullenia quinqueloba (Reuss) (Plate 4, Figure 9)}

Nonionina quinqueloba Reuss, 1851, Z. Deut. Geol. Ges., v. 3. Rather common at Sites 360, 362A, and 363, rare at Site 361 .

\section{Pullenia sp. 1}

(Plate 4, Figures 5, 6)

This indetermined species is present at all sites. Rather common at Sites 360, 363, and 364; rare at Sites 361 and 362A. Tabulated as Pullenia sp. in Table 3. 
Rectuvigerina elegans (Hantken)

Dimorphina elegans Hantken, 1875, Ungar. Geol. Anst. Mitt. Jb., v. 4, p. 63, pl. 7, fig. 9. Rare at Site 361 .

\section{Remesella varians (Glaessner) \\ (Plate 1, Figure 14)}

Textulariella ? varians Glaessner, 1937, Probl. Paleontol., v. 2, 3, p. 366 , pl. 2, fig. 15. Rather common at Site 363.

Siphotextularia finlayi Hornibrook

Siphotextularia finlayi Hornibrook, 1961, New Zealand Geol. Surv. Paleontol. Bull., v. 34 (1), p. 23, pl. 2, fig. 21, 26. Rare at Site 361 .

\section{Spiroplectammina dentata (Alth)}

(Plate 1, Figure 4)

Textularia dentata Alth, 1850, Natw. Abh., Wien, v. 3, p. 262, pl. 13, fig. 13. Scarce at Sites 363 and 364.

\section{Spiroplectammina excolata (Cushman)}

Textularia excolata Cushman, 1926, Am. Assoc. Petrol. Geol. Bull., v. 10, p. 585, pl. 15, fig. 9. Rare at Site 363.

\section{Stainforthia ryani Proto Decima and Bolli, n. sp.} (Plate 2, Figures 17, 18)

Test small, stout, spindle-shaped. Circular in transverse section, tapering at both ends, with the greatest width in about the middle of the test. Aperture loop-shaped, bordered by a narrow lip, terminal. Wall hyaline, finely perforate, surface smooth. Sutures distinct, depressed. Chambers inflated, overlapping, early stage triserial, biserial in the adult portion. The initial pointed end can have a distinct spine. Rather common in the middle Eocene of DSDP Leg 40 Site 361.

Dimension of holotype: (Plate 2, Figure 17): $0.35 \mathrm{~mm}$.

Dimension of figured paratype: (Plate 2, Figure 18): $0.3 \mathrm{~mm}$.

Type locality: Cape Basin, South Atlantic, $35^{\circ} 04^{\prime} \mathrm{S}, 15^{\circ} 27^{\prime} \mathrm{E}$.

Type sample: DSDP Leg 40 Site 361 , Core 6, core catcher.

Type stratum: Middle Eocene, Discoaster sublodoensis Zone.

Name: The species is named for William B. F. Ryan, DSDP Leg

40 co-chief scientist; Lamont-Doherty Geological Observatory, Palisades, New York.

Depository: Natural History Museum, Basel, No. C 33864 (Holotype), C 33865 (Paratype).

\section{Stilostomella cf. consobrina (d'Orbigny)}

cf. Dentalina consobrina d'Orbigny, 1846, Foram Bassin Tert. Vienne, p. 46 , pl. 2, fig. 1-3. Rare at Site 361 .

\section{Stilostomella curvatura (Cushman)}

(Plate 2, Figures 8, 9)

Ellipsonodosaria curvatura Cushman, 1939, Contrib. Cushman Lab. Foram. Res., v. 15, p. 71, pl. 12, fig. 6. Rare at Site 363.

\section{Stilostomella nuttalli (Cushman and Jarvis)}

(Plate 2, Figures 6, 7)

Ellipsonodosaria nuttalli Cushman and Jarvis, 1934, Contrib. Cushman Lab. Foram. Res., v. 10, p. 72, pl. 10, fig. 6. Rather common at Sites 360,361 , and 363.

\section{Stilostomella nuttalli aculeata (Cushman and Renz)}

Ellipsonodosaria nuttalli Cushman and Jarvis var. aculeata Cushman and Renz, 1948, Cushman Lab. Foram. Res., Spec. Publ., 24, p. 32 , pl. 6, fig. 10. Rare at Site 361 .

\section{Stilostomella nuttalli gracillima (Cushman and Jarvis)}

Ellipsonodosaria nuttalli Cushman and Jarvis var. gracillima Cushman and Jarvis, 1934, Contrib. Cushman Lab. Foram. Res., v. 10 , p. 72 , pl. 10, fig. 7. Rather common at sites 360 and 363 ; rare at Sites 361 and 364 .

\section{Stilostomella cf. stachei (Chapman)}

cf. Nodosaria stachei Chapman, 1926, New Zealand, Geol. Surv. Paleontol. Bull., v. 11, p. 53, pl. 3, fig. 16. Rare at Site 361.

\section{Stilostomella subspinosa (Cushman)}

Ellipsonodosaria subspinosa Cushman, 1943, Contrib. Cushman Lab. Foram. Res., v. 19, p. 92, pl. 16, fig. 6, 7. Rather common at Sites 360 and 363; rare at Sites 361 and $362 \mathrm{~A}$.

\section{Stilostomella verneuili (d’Orbigny)}

Dentalina verneuili d'Orbigny, 1846, Foram. Bassin Tert. Vienne, p. 48, pl. 2, fig. 7, 8. Rather common at Site 360; rare at Sites 361 and 363 .

Tappanina selmensis (Cushman)

(Plate 2, Figure 5)

Bolivinita selmensis Cushman, 1933, Contrib. Cushman Lab. Foram. Res., v. 9, p. 58, pl. 7, fig. 3, 4. Common in a single lower Eocene sample of Site 361 .

\section{Tritaxia amorpha (Cushman)}

Clavulina amorpha Cushman, 1926, Am. Assoc. Petrol. Geol. Bull., v. 10 , p. 589 , pl. 17 , fig. 5 . Rare at Site 364.

\section{Tritaxia aspera (Cushman)}

Clavulina trilatera Cushman, var. aspera Cushman, 1926, Am. Assoc. Petrol. Geol. Bull., v. 10, p. 589, pl. 17, fig. 3. Rare at Site 363.

\section{Tritaxia trilatera (Cushman)}

Clavulina trilatera Cushman, 1926, Am. Assoc. Petrol. Geol. Bull., v. 10 , p. 588 , pl. 17 , fig. 2 . Scarce at Site 363.

\section{Tritaxilina cubensis Cushman and Bermudez}

Tritaxilina cubensis Cushman and Bermudez, 1936, Contrib. Cushman Lab. Foram. Res., v. 12, pl. 10, fig. 25, 26. Rare at Site 363.

\section{Tritaxilina pupa (Guembel)}

Gaudryina pupa Guembel, 1868, Abh. K. Bayer. Akad. Wiss., II cl., v. 19, II Abt., p. 602, pl. 18, fig. 8-15. Rare at Sites 360, 362A, and 364 .

\section{Uvigerina acutocostata (Hagn)}

Hopkinsina acuto-costata Hagn, 1956, Palaeontographica, Abt. A, v. 107 , p. 151 , pl. 13, fig. 13. Rare at Site $362 \mathrm{~A}$.

\section{Uvigerina biscrialis Cushman and Edwards}

Uvigerina biscrialis Cushman and Edwards, 1937, Contrib. Cushman Lab. Foram. Res., v. 13, p. 59, pl. 8, fig. 11, 12. Rare at Site $362 \mathrm{~A}$.

\section{Uvigerina chirana Cushman and Stone}

(Plate 2, Figure 22)

Uvigerina chirana Cushman and Stone, 1947, Cushman Lab. Foram. Res., Spec. Publ., 20, p. 17, pl. 2, fig. 25. Scarce at Site 360.

\section{Uvigerina elongata Cole \\ (Plate 2, Figures 20, 21)}

Uvigerina elongata Cole, 1927, Am. Paleontol. Bull, v. 14, p. 26 , pl. 4, fig. 2, 3. Scarce at Sites 361 and 363.

\section{Uvigerina cf. eocaena Guembel}

cf. Uvigerina eocaena Guembel, 1868, Abh. k. Bayer. Akad. Wiss., II cl., v. 10, II Abt., p. 645 , pl. 2, fig. 78 . Rare at Site 361 .

\section{Uvigerina aff. gallowayi Cushman}

(Plate 2, Figure 23)

Uvigerina gallowayi Cushman, 1929. Contrib. Cushman Lab. Foram. Res., v. 5 , p. 94 , pl. 13, fig. 33,34 . Rare at Site 360.

\section{Uvigerina spinicostata Cushman and Jarvis} (Plate 2, Figure 19)

Uvigerina spinicostata Cushman and Jarvis, 1929, Contrib. Cushman Lab. Foram. Res., v. 5, p. 12, pl. 3, fig. 9, 10. Rather common at Sites 360 and 363. 
Vulvulina haeringensis (Guembel)

$$
\text { (Plate 1, Figure 5) }
$$

Venilina haeringensis Guembel, 1868, Abh. K. Bayer, Akad. Wiss., II cl. v. 10, II Abt., p. 649, pl. 2, fig. 84. Rather common at Sites $360,362 \mathrm{~A}$, and 363 .

\section{DEPOSITORY OF SPECIMENS}

The specimens figured on Plates 1 to 6 are deposited at the Museum of Natural History, Basel, under the Numbers C 33829 to 33948 .

\section{ACKNOWLEDGMENTS}

The authors wish to thank J.P. Beckmann for discussing general problems, and faunal distributions and their biostratigraphic significance, and for reading the manuscript.

\section{REFERENCES}

Beckmann, J. P., 1953. Die Foraminiferen der Oceanic Formation (Eocaen-Oligocaen) von Barbados, K1. Antillen: Ecolog. Geol. Helv. v. 46, p. 301-412. , 1960. Distribution of benthonic foraminifera at the Cretaceous Tertiary boundary of Trinidad (West Indies), 21st Int. Geol. Congr. Rept. 5, p. 57-69.

Berggren, W. A. and Aubert, J., 1975. Paleocene benthonic foraminiferal biostratigraphy, paleobiogeography and paleoecology of Atlantic-Tethyan regions: Midway-type fauna: Paleogeogr., Paleoclimat., Paleoecol., v. 18, p. 73192.

Braga, Gp., De Biase, R., Grünig, A., and Proto Decima, F., 1975. Foraminiferi bentonici del Paleocene ed Eocene della sezione di Possagno: Schweiz. Pal. Abh., v. 97, p. $85-110,187-199$.

Cushman, J. A., 1926. The foraminifera of the Velasco Shale of the Tampico embayment: Am. Assoc. Petrol. Geol. Bull., v. 10, p. 581-612.
1928. Cretaceous foraminifera from Trinidad: Contrib. Cushman Lab. Foram. Res., v. 4, p. 85-103.

Cushman, J. A. and Renz, H. H., 1946. The foraminiferal fauna of the Lizard Springs Formation of Trinidad, British West Indies: Cushman Lab. Foram. Res., Spec. Publ., no. 18 , p. 1-48.

1948. Eocene foraminifera of the Navet and

Hospital Hill formations of Trinidad, B.W.I.: Cushman Lab. Foram. Res., Spec. Publ., no. 24, p. 1-42.

Cushman, J. A. and Stainforth, R. M., 1945. The foraminifera of the Cipero Marl Formation of Trinidad, British West Indies: Cushman Lab. Foram. Res., Spec. Publ., no. 14, p. 1-91.

Glaessner, M. F., 1937. Studien uber Foraminiferan aus der Kreide und dem Tertiär des Kaukasus. I. Die Foraminiferen der ältesten Tertiärschichten des Nordwestkaukasus: Probl. Paleont., v. 2, 3, p. 309-408.

Gohrbandt, K., 1961. Die Kleinforaminiferenfauna des obereozänen Anteils der Reingruber Serie bei Bruderndorf (Bezirk Kronenburg, Niederösterreich): Mitt. Geol. Ges. Wien, v. 54 , p. 55-145.

Hagn, H. 1954. Some Eocene Foraminifera from the Bavarian Alps and adjacent areas: Contrib. Cushman Found. Foram. Res., v. 5, p. 14-20. 1956. Geologische und Paläontologische Untersuchungen im Tertiär des Monte Brione und seiner Umgebung (Gardasee, Oberitalien): Paläontographica, Abt. A, v. 107, p. 67-210.

Hillebrandt, A., 1962. Das Paleozän und seine Foraminiferenfauna im Becken von Reichenhall und Salzburg: Bayer. Akad. Wiss., math.-natw. Cl. Abh., (N.F.), v. 108, p. 1-182.

Hornibrook, N., de B., 1961. Tertiary foraminifera from Oamaru District (N.Z.): New Zealand Geol. Surv. Paleontol. Bull., v. 34, p. 1-192.

Nuttall, W. L., 1930. Eocene foraminifera from Mexico: J. Paleontol., v. 4, p. 271-293.

White M. P., 1928-1929. Some index foraminifera of the Tampico Embayment area of Mexico: J. Paleontol., v. 2, p. $177-215,280-317$; v. 3 , p. $30-58$. 
PLATE 1

Figure 1

Figure 2

Figure 3

Figure 4

Figure 5

Figure 6

Figure 7

Figure 8

Figure 9

Figure 10

Figure 11

Figure 12

Figure 13

Figure 14

Figure 15

Figure 16

Figure 17

Figure 18

Figure 19

Figure 20
Ammodiscus glabratus Cushman and Jarvis. $\times 40$; Site 360, Sample 45-3, 83-85 cm; C 33829.

Glomospira charoides (Jones and Parker). $\times 100$; Site 360, Sample 49, CC; C 33830 .

Bolivinopsis spectabilis (Grzybowski). $\times 40$; Site 360, Sample 36, CC; C 33831.

Spiroplectammina dentata (Alth).

$\times 60$; Site 364, Sample 10, CC; C 33832.

Vulvulina haeringensis (Guembel).

$\times 30$; Site 363, Sample 2, CC; C 33833.

Gaudryina pyramidata Cushman.

$\times 35$; Site 363, Sample 16, CC; C 33834.

Gaudryina pseudocollinsi Cushman and Stainforth. $\times 40$; Site 360, Sample 34-1, 89-91 cm; C 33835.

Tritaxia trilatera (Cushman).

$\times 50$; Site 363, Sample 16, CC; C 33836.

Karreriella subglabra (Guembel).

$\times 70$; Site 360, Sample 40, CC; C 33837.

Dorothia beloides Hillebrandt. $\times 80$; Site 364, Sample 10-5, 58-60 cm; C 33838.

Clavulina aff. anglica (Cushman).

$\times 50$; Site 360, Sample 36, CC; C 33839.

Clavulina cocoaensis (Cushman).

$\times 40$; Site 362 , Sample $12-1,108-110 \mathrm{~cm}$; C 33840 .

Plectina dalmatina (Schubert).

$\times 50$; Site 362A, Sample 9, CC; C 33841.

Remesella varians (Glaessner).

$\times 60$; Site 363, Sample 16, CC; C 33842 .

Marssonella nacataensis (White).

$\times 40$; Site 363, Sample 15, CC; C 33843.

Crysalogonium tenuicostatum Cushman and Bermudez.

×40; Site 361, Sample 8, CC; C 33844.

Orthomorphina rohri (Cushman and Stainforth). $\times 80$; Site 363, Sample 16, CC; C 33845.

Neoflabellina semireticulata (Cushman and Jarvis). $\times 60$; Site 363, Sample 16, CC; C 33846.

Praebulimina beaumonti (Cushman and Renz). $\times 60$; Site 363, Sample 16, CC; C 33847.

Praebulimina grata (Parker and Bermudez). $\times 100$; Site 363, Sample 2, CC; C 33848. 


\section{PLATE 1}

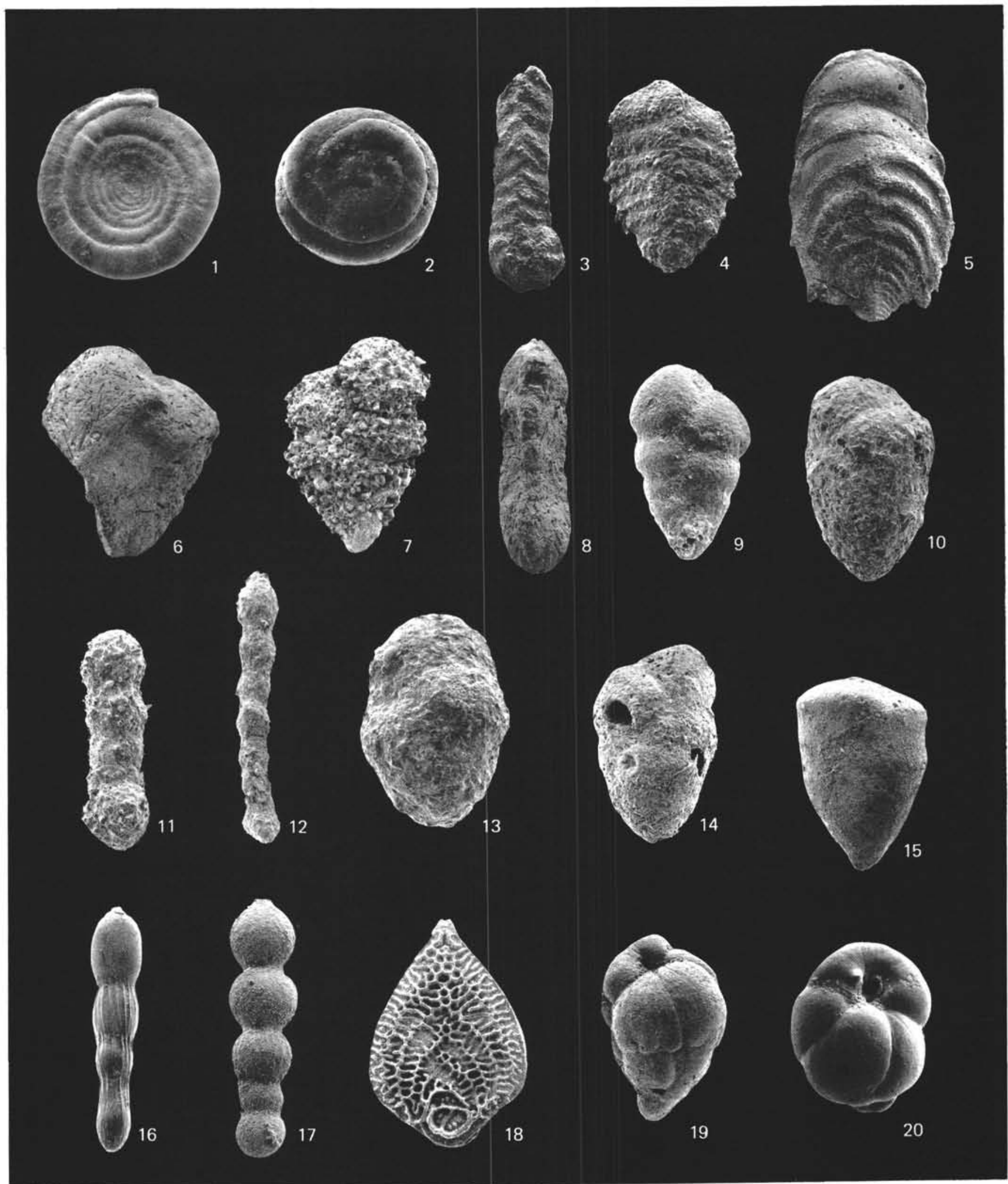




\section{PLATE 2}

Figure 1 Coryphostoma cf. limonense (Cushman). $\times 80$; Site 363, Sample 16, CC; C 33849.

Figure 2 Bolivina antegressa Subbotina. $\times 140$; Site 361, Sample 2, CC; C 33850.

Figure $3 \quad$ Loxostomoides dupuyi (Colom). $\times 50$; Site 361, Sample 6, CC; C 33851.

Figure 4 Detail of Figure 3. $\times 500$.

Figure 5 Tappanina selmensis (Cushman). $\times 140$; Site 361, Sample 8, CC; C 33852.

Figure 6 Stilostomella nuttalli (Cushman and Jarvis). $\times 12$; Site 364, Sample 302, 58-60 cm; C 33853 .

Figure 7 Stilostomella nuttalli (Cüshman and Jarvis). $\times 25$; Site 363, Sample 2, CC; C 33854.

Figure $8 \quad$ Stilostomella curvatura (Cushman). $\times 20$; Site 353, Sample 2, CC; C 33855.

Figure 9 Stilostomella curvatura (Cushman). $\times 30$; Site 353, Sample 2, CC; C 33856.

Figure 10 Bulimina alazanensis Cushman. $\times 80$; Site 363, Sample 9-3, 73-75 cm; C 33857.

Figure 11 Bulimina impendens Parker and Bermudez. $\times 100$; Site 360, Sample 32, CC; C 33858.

Figure 12 Bulimina impendens Parker and Bermudez. $\times 100$; Site 361, Sample 6, CC; C 33859.

Figure 13 Bulimina jarvisi Cushman and Parker. $\times 70$; Site 360, Sample 32-1, 107-110 cm; C 33860 .

Figure $14 \quad$ Bulimina semicostata Nuttall. $\times 100$; Site 361, Sample 6, CC; C 33861 .

Figure 15 Bulimina trinitatensis Cushman and Jarvis. Specimen without costae. $\times 100$; Site 363, Sample 17, CC; C 33862.

Figure 16 Bulimina trinitatensis Cushman and Jarvis. $\times 80$; Site 363, Sample 16, CC; C 33863.

Figure 17 Stainforthia ryani Proto Decima and Bolli, n. sp., Holotype.

$\times 120$; Site 361, Sample 6, CC; C 33864.

Figure 18 Stainforthia ryani Proto Decima and Bolli, n. sp., Paratype.

$\times 140$; Site 361, Sample 6, CC; C 33865.

Figure 19 Uvigerina spinicostata Cushman and Jarvis. $\times 100$; Site 363, Sample 5, CC; C 33866.

Figure $20 \quad$ Uvigerina elongata Cole. $\times 60$; Site 363, Sample 6, CC; C33867.

Figure $21 \quad$ Detail of Figure 20. $\times 250$.

Figure 22 Uvigerina chirana Cushman and Stone. $\times 70$; Site 360, Sample 42, CC; C 33868.

Figure 23 Uvigerina aff. gallowayi Cushman. $\times 80$; Site 360, Sample 27, CC; C 33869. 
PLATE 2
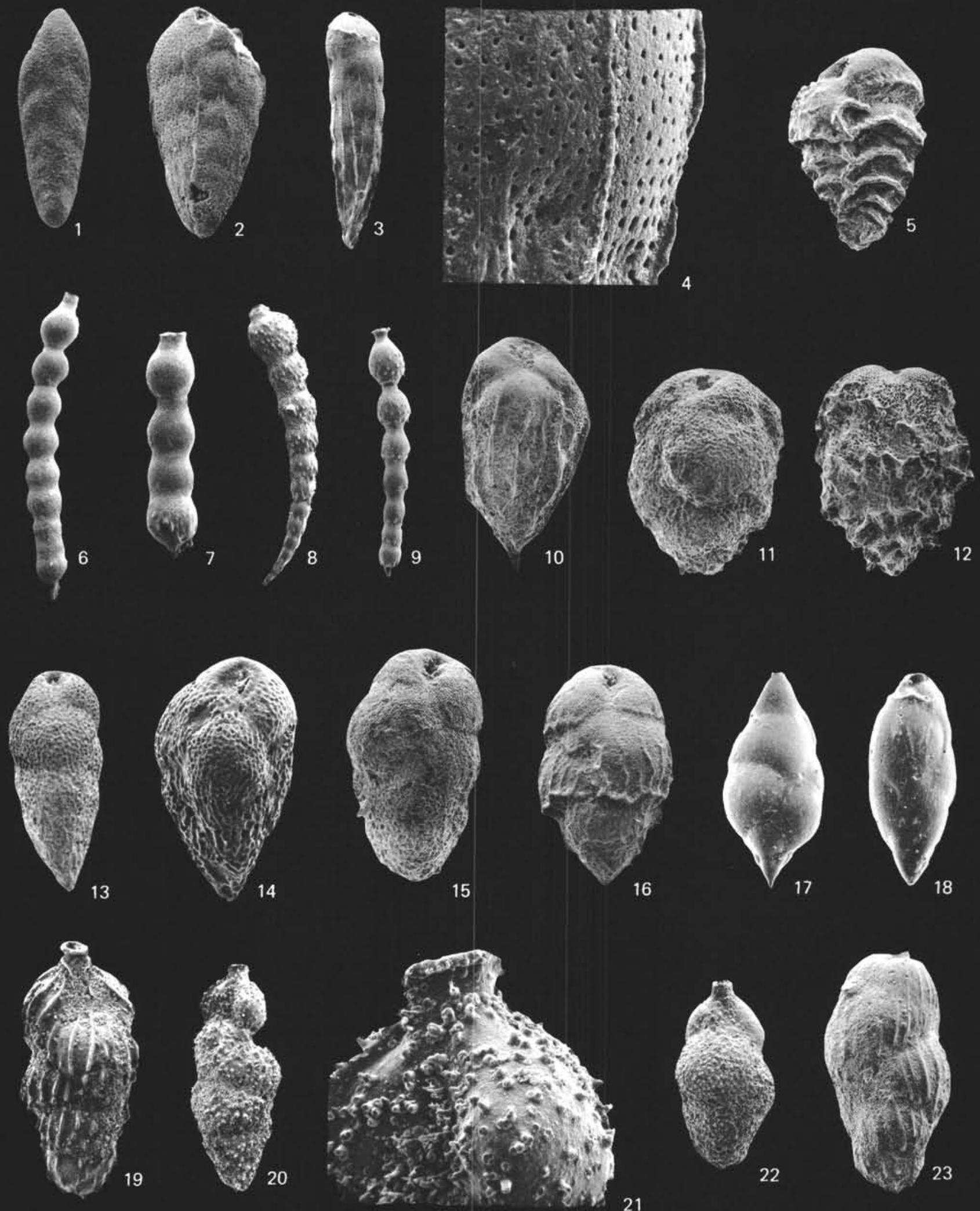
PLATE 3

Figure $1 \quad$ Nuttallides truempyi (Nuttall), spiral view. $\times 60$; Site 360 , Sample 50, CC; C 33870.

Figure 2 Nuttallides truempyi (Nuttall), umbilical view. $\times 50$; Site 360, Sample 50, CC; C 33871.

Figure $3 \quad$ Eponides lotus (Schwager), spiral view. $\times 80$; Site 363, Sample 16, CC; C 33872.

Figure $4 \quad$ Eponides lotus (Schwager), umbilical view. $\times 90$; Site 364, Sample 10, CC; C 33873.

Figure 5 Planulina ammophila (Guembel), spiral view. $\times 50$; Site 360, Sample 32-1, 107-110 cm; C 33874 .

Figure $6 \quad$ Planulina ammophila (Guembel), umbilical view. $\times 60$; Site 360, Sample 32-1, 107-110 cm; C 33875 .

Figure 7 Planulina renzi Cushman and Stainforth, spiral view.

X80; Site 360, Sample 29, CC; C 33876.

Figure $8 \quad$ Cibicides $\mathrm{sp}$. 1, spiral view. $\times 100$; Site 360, Sample 26, CC; C 33877.

Figure 9 Pleurostomella acuta Hantken. $\times 80$; Site 360, Sample 34, CC; C 33878.

Figure $10 \quad$ Pleurostomella alternans Schwager. $\times 40$; Site 363, Sample 2, CC; C 33879.

Figure 11 Pleurostomella incrassata Hankten. $\times 100$; Site 360, Sample 40, CC; C 33880 .

Figure 12 Bandyella beckmanni Proto Decima and Bolli, $\mathrm{n}$. sp., Holotype.

$\times 50$; Site 364, Sample 10-1, 58-60 cm; C 33881 .

Figure $13 \quad$ Pleurostomella nuttalli Cushman and Siegfus. $\times 50$; Site 360 , Sample 29, CC; C 33882.

Figure $14 \quad$ Ellipsodimorphina subcompacta Liebus. $\times 50$; Site 360, Sample 50, CC; C 33883.

Figure $15 \quad$ Ellipsoidina ellipsoides Seguenza. $\times 80$; Site 360, Sample 29-2, 73-75 cm; C 33884.

Figure 16 Ellipsoglandulina multicostata (Galloway and Morrey).

$\times 80$; Site 361, Sample 1, CC; C 33885.

Figure 17 Detail of Figure 16. $\times 500$.

Figure $18 \quad$ Nodosarella subnodosa (Guppy).

$\times 40$; Site 360, Sample 27-1, 56-58 cm; C 33886 .

Figure 19 Cassidulina havanensis Cushman and Bermudez. $\times 100$; Site 363, Sample 8, CC; C 33887.

Figure $20 \quad$ Arăgonia aragonensis (Nuttall). $\times 100$; Site 360, Sample 48, CC; C 33888.

Figure $21 \quad$ Aragonia velascoensis (Cushman). $\times 60$; Site 363, Sample 16, CC; C 33889.

Figure 22 Aragonia ouezzanensis (Rey). $\times 50$; Site 364, Sample 10-1, 58-60 cm; C 33890 .

Figure 23 Globocassidulina globosa (Hantken). $\times 100$; Site 361, Sample 1, CC; C 33891.

Figure 24 Globocassidulina globosa (Hantken). $\times 50$; Site 363, Sample 2, CC; C 33892. 
PLATE 3

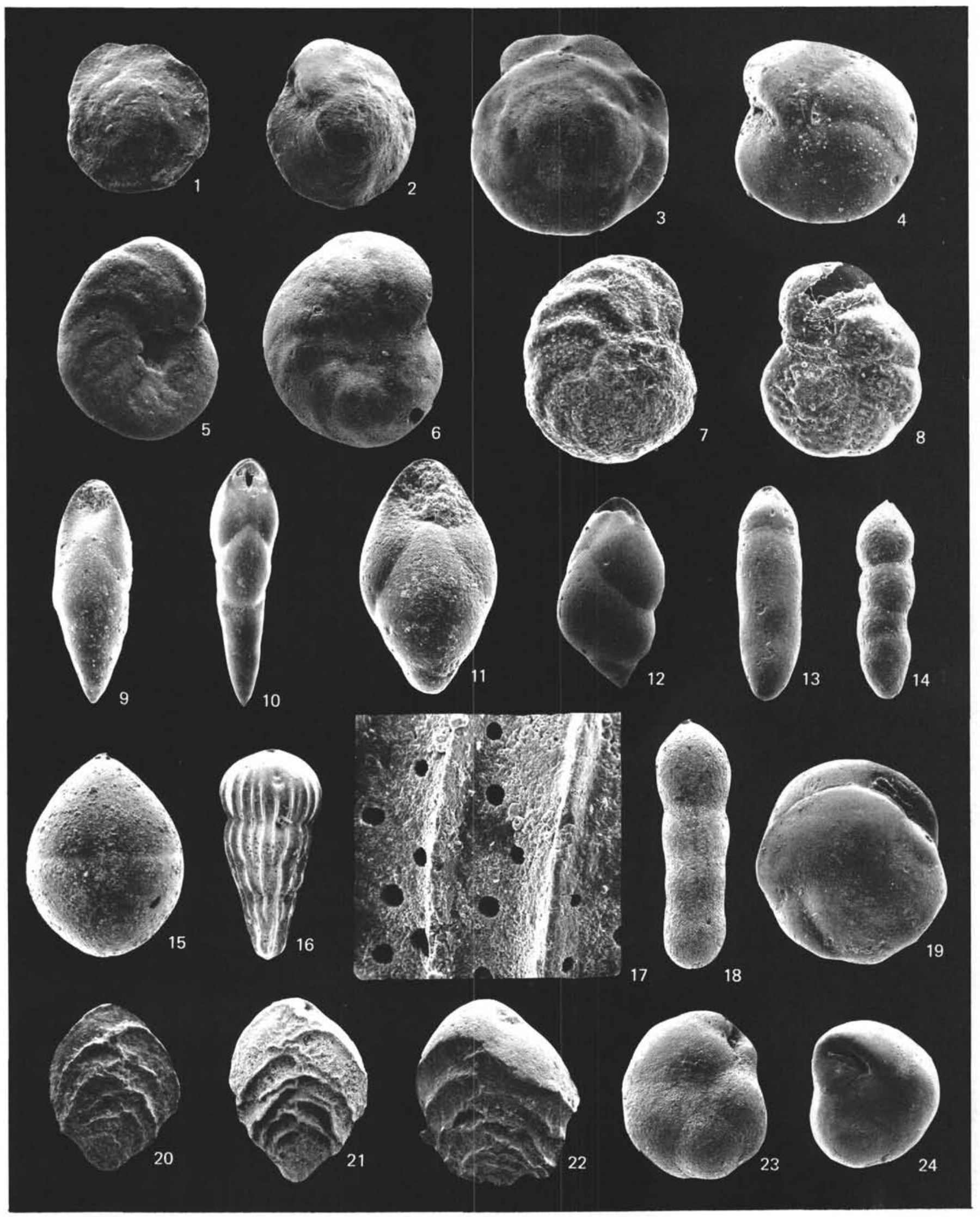




\section{PLATE 4}

Figure $1 \quad$ Nonion havanense Cushman and Bermudez. $\times 100$; Site 363, Sample 15-3, 58-60 cm; C 33893 .

Figure 2 Nonion havanense Cushman and Bermudez. $\times 100$; Site 363, Sample 15-3, 58-60 cm; C 33894 .

Figure 3 Pullenia coryelli White. $\times 100$; Site 363, Sample 16, CC; C 33895.

Figure $4 \quad$ Pullenia coryelli White. $\times 100$; Site 363, Sample 16, CC; C 33896.

Figure $5 \quad$ Pullenia sp. 1. $\times 100$; Site 363, Sample 6, CC; C 33897.

Figure $6 \quad$ Pullenia sp. 1. $\times 100$; Site 363, Sample 6, CC; C 33898.

Figure $7 \quad$ Pullenia eocenica Cushman and Siegfus. $\times 100$; Site 360, Sample 50, CC; C 33899.

Figure $8 \quad$ Pullenia eocenica Cushman and Siegfus. $\times 100$; Site 360, Sample 50, CC; C 33900.

Figure $9 \quad$ Pullenia quinqueloba (Reuss). $\times 120$; Site 363 , Sample 9-3, 82-84 cm; C 33901.

Figure $10 \quad$ Alabamina dissonata (Cushman and Renz), spiral view.

$\times 100$; Site 364, Sample 7, CC; C 33902.

Figure 11 Alabamina dissonata (Cushman and Renz), umbilical view.

$\times 100$; Site 364, Sample 7, CC; C 33903.

Figure 12 Gyroidinoides subangulata (Plummer), spiral view. $\times 80$; Site 362A, Sample 2, CC; C 33904.

Figure 13 Gyroidinoides planulata (Cushman and Renz), spiral view.

X80; Site 360, Sample 29, CC; CC 33905.

Figure 14 Gyroidinoides planulata (Cushman and Renz), umbilical view.

$\times 100$; Site 360, Sample 29, CC; C 33906.

Figure 15 Osangularia pteromphalia (Guembel), spiral view. $\times 50$; Site 363, Sample 10-4, 58-60 cm; C 33907.

Figure 16 Osangularia pteromphalia (Guembel), umbilical view.

$\times 50$; Site 363, Sample 10-4, 58-60 cm; C 33908.

Figure 17 Charltonina florealis (White), spiral view. $\times 40$; Site 363, Sample 15, CC; C 33909.

Figure 18 Charltonina florealis (White), umbilical view. $\times 35$; Site 363, Sample 15, CC; C 33910.

Figure 19 Gyroidinoides globosa (Hagenow), spiral view. $\times 60$; Site 363, Sample 15, CC; C 33911.

Figure $20 \quad$ Gyroidinoides globosa (Hagenow), apertural view. $\times 60$; Site 363, Sample 15, CC; C 33912. 

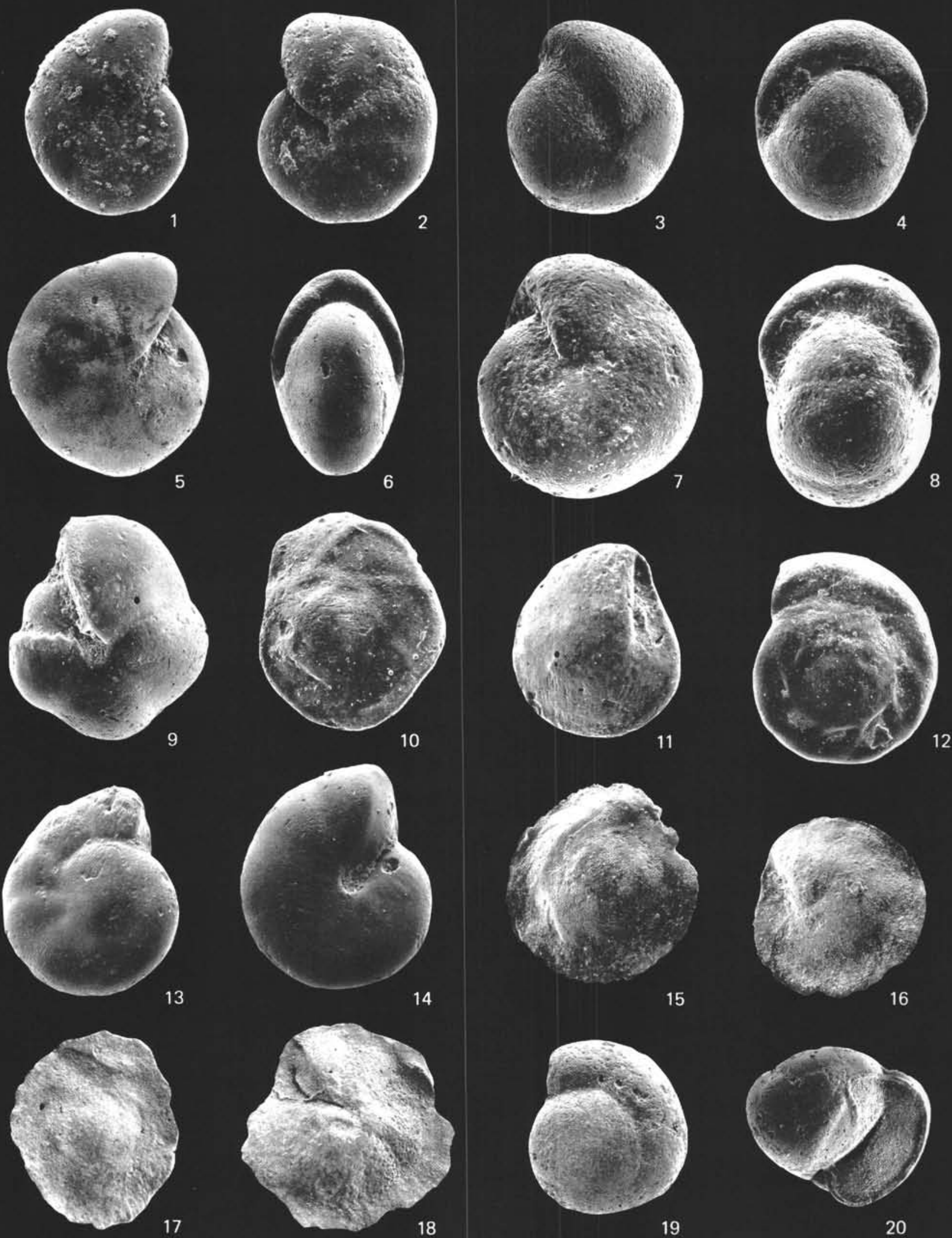


\section{PLATE 5}

Figure 1 Gyroidinoides soldanii (d'Orbigny), spiral view. $\times 100$; Site 363, Sample 4, CC; C 33913.

Figure 2 Gyroidinoides soldanii (d'Orbigny), spiral view. $\times 100$; Site 363, Sample 4, CC; C 33914.

Figure 3

Gyroidinoides octocamerata (Cushman and Hanna), spiral view.

$\times 70$; Site 360, Sample 35-2, 134-136 cm; C 33915 .

Figure 4

Gyroidinoides octocamerata (Cushman and Hanna), umbilical view.

$\times 70$; Site 360, Sample 35-2, 134-136 cm; C 33916.

Figure 5

Anomalina pompilioides semicribrata Beckmann, spiral view.

$\times 60$; Site 360, Sample 47-2 (bottom); C 33917.

Figure 6

Anomalina pompilioides semicribrata Beckmann, umbilical view.

$\times 60$; Site 360, Sample 47-2 (bottom); C 33918 .

Figure 7 Anomalina capitata (Guembel), spiral view. $\times 60$; Site 360 , Sample 47-2 (bottom); C 33919 .

Figure 8 Anomalina capitata (Guembel), umbilical view. $\times 60$; Site 360, Sample 47-2 (bottom); C 33920 .

Figure 9 Anomalina praeacuta Vasilenko, spiral view. $\times 120$; Site 361, Sample 8, CC; C 33921.

Figure $10 \quad$ Detail of Figure 9. $\times 500$.

Figure 11 Anomalina praeacuta Vasilenko, umbilical view. $\times 120$; Site 361, Sample 8, CC; C 33922.

Figure 12 Anomalina alazanensis spissiformis Cushman and Stainforth, spiral view.

$\times 70$; Site 360, Sample 27, CC; C 33923.

Figure 13 Anomalina alazanensis spissiformis Cushman and Stainforth, umbilical view.

$\times 70$; Site 360, Sample 27, CC; C 33924.

Figure 14 Anomalina? sp. 1, side view.

$\times 180$; Site 361, Sample 4, CC; C 33925.

Figure 15 Anomalina? sp. 1, umbilical view.

$\times 180$; Site 361, Sample 4, CC; C 33926.

Figure 16 Cibicidoides aff. cookei (Cushman and Garrett), spiral view.

$\times 60$; Site 363, Sample 9-3, 105-107 cm; C 33927.

Figure 17 Cibicidoides aff. cookei (Cushman and Garrett), umbilical view.

$\times 60$; Site 363, Sample 9-3, 105-107 cm; C 33928.

Figure 18 Cibicidoides alleni (Plummer), spiral view.

$\times 70$; Site 363, Sample 16, CC; C 33929.

Figure 19 Cibicidoides alleni (Plummer), umbilical view.

$\times 60$; Site 363, Sample 15-3, 58-60 cm; C 33930 . 

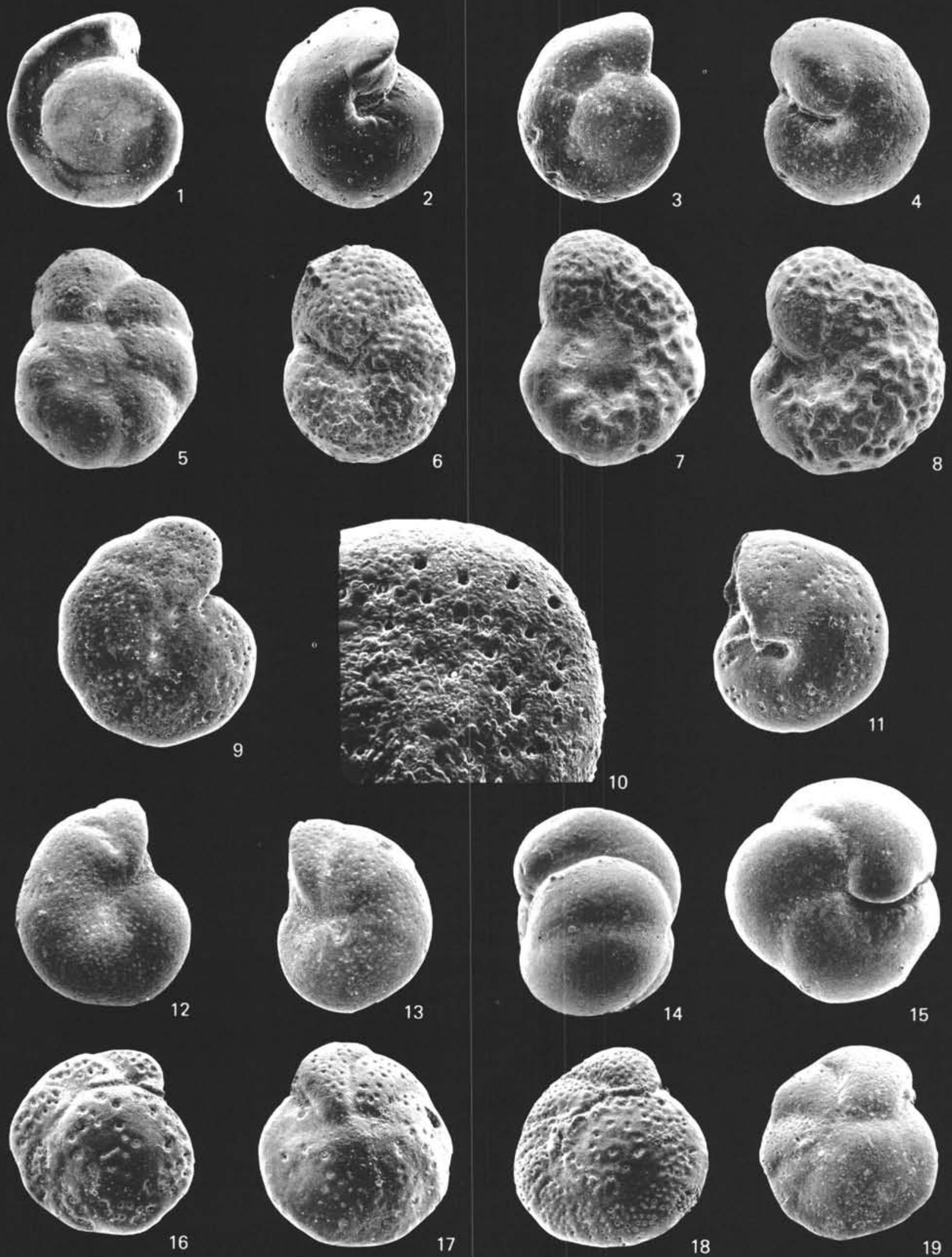


\section{PLATE 6}

Figure 1 Gavelinella dayi (White), spiral view. $\times 40$; Site 363, Sample 16, CC; C 33931.

Figure 2 Gavelinella dayi (White), umbilical view. ×40; Site 363, Sample 16, CC; C 33932.

Figure 3 Gavelinella beccariiformis (White), spiral view. $\times 60$; Site 363, Sample 16, CC; C 33933.

Figure 4 Gavelinella beccariiformis (White), umbilical view. $\times 60$; Site 363, Sample 16, CC; C 33934.

Figure 5 Gavelinella micra (Bermudez), spiral view. $\times 80$; Site 363, Sample 5, CC; C 33935.

Figure 6 Gavelinella micra (Bermudez), umbilical view. $\times 80$; Site 363, Sample 5, CC; C 33936.

Figure $7 \quad$ Gavelinella rubiginosa (Cushman), spiral view. $\times 80$; Site 363, Sample 15, CC; C 33937.

Figure $8 \quad$ Gavelinella rubiginosa (Cushman), umbilical view. $\times 60$; Site 363, Sample 15, CC; C 33938.

Figure 9 Heterolepa reussi (Silvestri), spiral view. $\times 50$; Site 363, Sample 2, CC; C 33939.

Figure 10 Detail of Figure 11, showing one of the large, conical-shaped parasite holes. On lower left is a group of the irregularly distributed pores. $\times 250$.

Figure $11 \quad$ Heterolepa reussi (Silvestri), umbilical view. $\times 50$; Site 363, Sample 2, CC; C 33940.

Figure 12 Heterolepa reussi (Silvestri),spiral view. view.

$\times 50$; Site 362A, Sample 4, CC; C 33941.

Figure 13 . Heterolepa pygmea (Hantken), spiral view. $\times 120$; Site 360, Sample 44, CC; C 33942.

Figure $14 \quad$ Heterolepa eocaena (Guembel), spiral view. $\times 30$; Site 363A, Sample 2, CC; C 33943.

Figure 15 Heterolepa eocaena (Guembel), umbilical view. $\times 45$; Site 362A, Sample 2, CC; C 33944.

Figure 16 Heterolepa grimsdalei (Nuttall), umbilical view. $\times 50$; Site 362A, Sample 7, CC; C 33945.

Figure 17 Heterolepa grimsdalei (Nuttall), spiral view. $\times 50$; Site 362A, Sample 7, CC; C 33946.

Figure 18 Heterolepa ungeriana (d'Orbigny), spiral view. $\times 50$; Site 363, Sample 10-4, 58-60 cm; C 33947.

Figure 19 Heterolepa ungeriana (d'Orbigny), spiral view. $\times 50$; Site 363, Sample 10-4, 58-60 cm; C 33948. 
PLATE 6
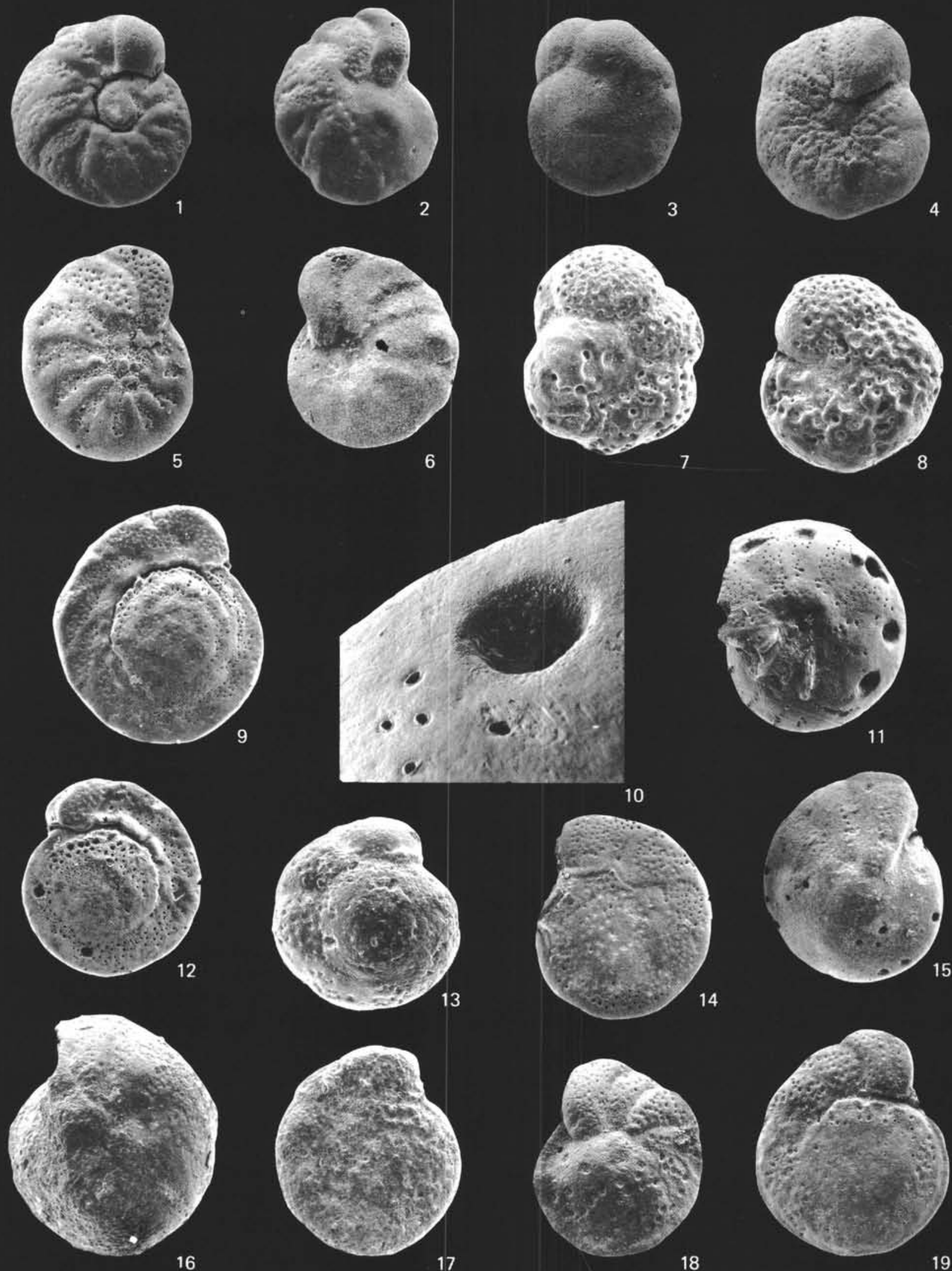